\title{
DIRITTI FONDAMENTALI E NUOVE TECNOLOGIE: UNA MAPPA DEL DIBATTITO ITALIANO
}

\section{FUNDAMENTAL RIGHTS AND DIGITAL REVOLUTION: THE ITALIAN DEBATE}

MARCO OLIVETTI ${ }^{1}$

RIASSUNTO: La rivoluzione digitale ha inciso profondamente sui diritti fondamentali. Essa ha aperto nuove prospettive per l'esercizio di diritti fondamentali già riconosciuti. Ha fatto emergere nuovi diritti fondamentali e nuovi limiti e nuove minacce ad essi. La cultura giuridica italiana ha concettualizzato queste sfide attorno ad una serie di temi: la libertà informatica, il diritto fondamentale di accesso ad Internet, il diritto all'oblio, il diritto a non essere oggetto di una decisione amministrativa totalmente automatizzata, i nuovi problemi della libertà di espressione. La Camera dei deputati ha elaborato una "dichiarazione dei diritti su Internet", senza efficacia normativa. E l'uso di Internet ha modificato profondamente il sistema politico. Questo saggio tenta un primo bilancio generale di questi problemi, che in generale sono ancora tutti aperti.

PAROLE CHIAVE: Diritti fondamentali; Internet; Algoritmi; Partecipazione politica.

\begin{abstract}
Fundamental rights have been profoundly affected by the digital revolution. New perspectives for the exercise of already recognized fundamental rights have been opened. New fundamental rights and new limits and new threats to them have emerged. The Italian legal culture has conceptualized these challenges around a series of topics: the "cyber" freedom, the fundamental right of access to the Internet, the right to be forgotten, the right not to be subject to a fully automated administrative decision, the new problems of freedom of expression. The Chamber of Deputies has drafted a not legally binding "declaration of rights on the Internet". And the use of the Internet has profoundly changed the political system. This essay tries to draw a first general assessment of these problems, which in general are still all in evolution.
\end{abstract}

Keywords: Fundamental rights; Internet; Algorithms; Political participation.

\footnotetext{
${ }^{1}$ Professore ordinario di Diritto costituzionale nel Dipartimento di Giurisprudenza, Economia, Scienze politiche e Lingue moderne della LUMSA di Roma. Email: m.olivetti65@gmail.com.
} 


\section{Premessa}

I diritti fondamentali strutturano l'azione sociale dell'uomo nelle sue diverse manifestazioni, proteggendo alcune sfere di azione umana dall'interferenza dello Stato e di altri soggetti ed abilitando il loro titolare ad accedere a prestazioni di vario tipo e a partecipare alla formazione della volontà collettiva. Le innovazioni tecnologiche che incidono sui comportamenti umani lanciano nuove sfide ai diritti fondamentali in quanto meccanismi di tutela dell'autonomia individuale e collettiva rispetto allo Stato e agli altri poteri, pubblici e privati. Del resto, questa correlazione non è una novità degli ultimi decenni ed è corretto osservare che «la pressione dell'innovazione tecnologica sul sistema giuridico è ... una costante storica» ${ }^{2}$.

Dai tempi della rivoluzione industriale, le «nuove» tecnologie hanno progressivamente investito ogni aspetto dell'organizzazione sociale ${ }^{3}$. La rivoluzione informatica, nelle sue successive ondate, ha inevitabilmente inciso da un lato sulla gamma delle azioni umane e sull'organizzazione della società civile, e dall'altro sull'organizzazione e sull'azione dello Stato e dei poteri pubblici ${ }^{4}$, influenzando, di conseguenza, anche i diritti fondamentali. In particolare, Internet - i cui utenti sono cresciuti dai 120 milioni del 1997 ai 3 miliardi nel 2013 e agli oltre 4 miliardi nel $2018^{5}$ - ha generato nuove opportunità per lo sviluppo della personalità individuale e per l'azione collettiva e, al tempo stesso, ha posto nuovi problemi di tutela della dignità, della libertà e dell'autonomia della persona. Anche l'apparizione e la straordinaria diffusione dei social network ha aperto nuove opportunità e ha fatto emergere nuove minacce per gli esseri umani e per i loro diritti.

Malgrado l'autonomia del cyberspazio o del mondo «virtuale», che è certamente portatore di codici di comportamento da esso stesso generati (ed intrinsecamente connessi con la sua dimensione tecnica), la pretesa estrema, avanzata da alcuni operatori di tale mondo, secondo la quale esso sarebbe portatore di una normatività autonoma (una sorta di lex informatica, che richiamerebbe la ben nota lex mercatoria), o addirittura di una rivendicazione (anarchica) di esenzione

\footnotetext{
${ }^{2}$ M. Betzu, Regolare Internet, Giappichelli, Torino, 2012, p. 15.

${ }^{3}$ Per un quadro generale dei problemi qui trattati si v. anche E. Bertolini, V. Lubello, O. Pollicino, Internet: regole e tutela dei diritti fondamentali, Aracne, Roma, 2015; O. Pollicino, T.E. Frosini, E. Apa, M. Bassini (a cura di), Diritti e libertà in Internet, Mondadori, Milano, 2017; E. Falletti, I diritti fondamentali su Internet. Libertà di espressione, privacy, copyright, II ed., Exeo, Brugine, 2017.

${ }^{4}$ Per un panorama descrittivo degli impatti della rivoluzione digitale sull'organizzazione politica si v. L. Casini, Lo Stato nell'era di Google, in Rivista trimestrale di diritto pubblico, 2019, n. 4, p. 1111-1148. Distinta dal tema trattato in questo saggio, ma connessa con esso, è la questione dell'impatto della rivoluzione digitale sul diritto amministrativo. Su questo tema si v. il panorama di diritto comparato proposto da J.-B. Auby, Il diritto amministrativo di fronte alle sfide digitali, in Le Istituzioni del Federalismo, 2019, n. 3, p. 619-641, nonché uno scorcio del dibattito italiano nello stesso numero della rivista in cui tale saggio è pubblicato.

${ }^{5}$ Si v. Global Digital 2019 , in https://wearesocial.com/global-digital-report-2019.
} 
dalla normatività generata dalle istituzioni del mondo «reale» ${ }^{6}$, si rivela insostenibile, proprio in quanto lo stesso fenomeno di Internet presuppone una serie di meccanismi regolativi, che rendono possibili le attività svolte mediante esso $^{7}$. Internet e il diritto non sono due entità mutuamente estranee. E la stessa configurazione di Internet come un ordinamento giuridico autonomo (e distinto dagli ordinamenti statali) ${ }^{8}$, o come ordinamento speciale ${ }^{9}$ se non vuole limitarsi a sottolineare da un lato le specificità di questo fenomeno e dall'altro la rivendicazione di spazi di libertà, tutelati anzitutto attraverso il ricorso al diritto privato, appare più una suggestione che una ricostruzione giuridica logicamente sostenibile. Certo, la natura del world wide web tende a sfuggire al potere regolativo statale e le norme giuridiche che intervengono in materia devono interagire con altre regole aventi natura economica, di costume e soprattutto tecnica (la Lex informatica o Code, appunto). Ma quasi mai il diritto, quando interviene a regolare una materia di qualsiasi tipo può sfuggire ad interazioni paragonabili a queste, anche se di tipo diverso caso per caso, cioè a regole sociali, economiche, tecniche ${ }^{10}$. In ogni caso l'analisi dell'ordinamento giuridico italiano conferma che il fenomeno Internet è oggetto di una regolazione legislativa, adottata talora in attuazione di direttive europee, talaltra in sintonia con le sedi internazionali di regolazione della Rete $^{11}$.

${ }^{6}$ Forse la formulazione più estrema di questa posizione si può trovare in J.P. Barlow, Declaration of independence of Cyberspace, in

www.erf.org/pub/publications/John Perry Barlow/barlow 0296.declaration. Nella stessa direzione si sono mossi argomenti di altri studiosi statunitensi, come D.R. Johnson, D.G. Post, Law And Borders: The Rise of Law in Cyberspace, in Stanford Law Review 48 (1996), p. 1367 ss. Si v. anche J.R. Reidenberg, Lex Informatica: The Formulation of Information Policy Rules Through Technology, in Texas Law Review, 1998, p. 553 ss. Si v. anche T.W. Goldsmith, Who Controls the Internet? Illusions of a Bordeless World, Oxford University Press, New York, 2006.

${ }^{7} \mathrm{Si}$ v. vari esempi in C.R. Sunstein, \#Republic. La democrazia nell'epoca dei social media, Il Mulino, Bologna, 2018, p. 224 ss.

${ }^{8}$ Su cui v. alcuni argomenti in T.E. Frosini, Internet come ordinamento giuridico, in M. Nisticò, P. Passaglia (a cura di), Internet e Costituzione, Giappichelli, Torino, 2014, p. 57-59.

${ }^{9}$ M. Betzu, Regolare Internet, cit., p. 22 ss.

${ }^{10} \mathrm{Mi}$ paiono pertanto eccessive le sottolineature di M. Betzu, Regolare Internet, cit., p. 22 ss. circa l'atipicità del rapporto fra internet e diritto.

${ }^{11}$ E' il caso di Registro.it, a cui spetta l'assegnazione del domain name. Sui diversi profili della governance di Internet, si v. B. Carotti, L'ICANN e la governance di Internet, in Rivista trimestrale di diritto pubblico, 2007, n. 3, p. 683 ss.; L. Koletsou, K. Komaitis, E. Maganaris, The Role of ICANN in Internet Governance: Friend or Foe?, in European Review of Public Law, 2006, n. 4, p. 1346 ss.; P. Costanzo, Aspetti evolutivi del regime giuridico di Internet, in Il diritto dell'informazione $e$ dell'informatica, n. 6, 1996, n. 6, p. 831 ss.; P. Costanzo, Internet (diritto pubblico), in Digesto delle discipline pubblicistiche, IV ed. Aggiornamento, Utet, Torino 2000, 347 ss.; D. De Grazia, L'Internet Governance tra tecnica, politica e diritto, in Informatica e diritto, 2009, n. 1, p. 29 ss.; AA.VV., I problemi giuridici di Internet, a cura di E. Tosi, Giuffrè, Milano, 1999; G. Pascuzzi, Il diritto dell'era digitale. Tecnologie informatiche e regole privatistiche, il Mulino, Bologna, 2002. 
Per oltre un quarto di secolo, il bilancio etico-politico delle varie fasi della rivoluzione informatica è sembrato largamente positivo ${ }^{12}$, nel senso che le nuove opportunità sono sembrate largamente prevalenti sui rischi e sulle minacce per $\mathrm{i}$ diritti della persona e per le istituzioni della democrazia liberale. L'ultimo quinquennio, tuttavia, ha fatto emergere una diversa percezione dei problemi e le valutazioni pessimistiche si sono notevolmente rafforzate rispetto a quelle ottimistiche. Quest'ultimo trend sollecita ulteriormente lo sviluppo di un discorso sul rapporto fra i diritti fondamentali e le nuove tecnologie, che era ovviamente emerso già nella fase precedente, nella quale Internet era apparso per lo più come uno strumento per la piena realizzazione delle «Magnifiche sorti e progressive» del genere umano ${ }^{13}$, $\mathrm{o}$, se si preferisce, come un poderoso strumento di liberazione. $\mathrm{Ma}$, con l'emersione in primo piano dei lati oscuri della rivoluzione digitale, all'esigenza di riconoscere nuovi diritti fondamentali si affianca quella di inquadrarli in nuovi limiti, sviluppando la ben nota massima secondo la quale nessun diritto fondamentale è assoluto e privo di limiti ${ }^{14}$.

\section{Quattro forme di impatto delle nuove tecnologie sui diritti fondamentali}

«La pressione che il contenuto dei diritti subisce per effetto del dinamismo tecnologico è un dato pressoché generalmente condiviso» ${ }^{15}$. In generale, si può

${ }^{12}$ B. Thieulin, Gouverner à l'heure de la révolution des pouvoirs, in Pouvoirs, n. 164 (2018), p. 19 ha notato, ad es., che «la révolution numerique ne saurait se réduire à une simple révolution technique. C'est avant tout une révolution des pouvoirs. Le numérique donne de nouveaux moyens et de nouvelles capacités d'action à ceux qui n'en avaient pas».

${ }^{13} \mathrm{Si}$ riprende qui la formula (celebre nella letteratura italiana) utilizzata da G. Leopardi, Canto XXXIV - La ginestra, o fiore del deserto, in Canti.

${ }^{14}$ Com'è noto, nella giurisprudenza costituzionale italiana questa massima, di importanza essenziale, malgrado talvolta se ne abusi, è stata formulata nella sent. n. 85/2013 della Corte costituzionale: «La Costituzione italiana, come le altre Costituzioni democratiche e pluraliste contemporanee, richiede un continuo e vicendevole bilanciamento tra princìi e diritti fondamentali, senza pretese di assolutezza per nessuno di essi. La qualificazione come "primari" dei valori dell'ambiente e della salute significa pertanto che gli stessi non possono essere sacrificati ad altri interessi, ancorché costituzionalmente tutelati, non già che gli stessi siano posti alla sommità di un ordine gerarchico assoluto». «tutti i diritti fondamentali tutelati dalla Costituzione si trovano in rapporto di integrazione reciproca e non è possibile pertanto individuare uno di essi che abbia la prevalenza assoluta sugli altri. La tutela deve essere sempre "sistemica e non frazionata in una serie di norme non coordinate ed in potenziale conflitto tra loro" (sent. n. 264/2012). Se così non fosse, si verificherebbe l'illimitata espansione di uno dei diritti, che diverrebbe "tiranno" nei confronti delle altre situazioni giuridiche costituzionalmente riconosciute e protette, che costituiscono, nel loro insieme, espressione della dignità della persona».

${ }^{15} \mathrm{M}$. Betzu, Regolare Internet, cit., p. 18. Per un quadro generale sulle sfide poste dall'era digitale ai diritti fondamentali si v. A. Cerri, Telecomunicazioni e diritti fondamentali in Il diritto dell'informazione e dell'informatica, 1996, n. 6, p. 785 ss.; G. Corasaniti, Diritti nella rete. Valori umani, regole, interazione tecnologica globale, F. Angeli, Milano, 2006; T.E. Frosini, Tecnologie e libertà costituzionali, in Il diritto dell'informazione e dell'informatica, 2003, n. 3, p. 487 ss., nonché in Scritti in memoria di Livio Paladin, 
osservare che Internet ha da un lato aperto la via alla possibilità di svolgere attività umane del tutto nuove, non configurabili nel mondo reale, e dall'altro ha configurato nuove modalità per svolgere attività umane che in precedenza venivano svolte in altri modi. L'estensione della sfera di azione dei diritti fondamentali alle attività umane svolte su o mediante Internet è certo più agevole in questo secondo caso, dato che il giurista può muoversi con la tecnica dell'interpretazione estensiva delle norme sui diritti fondamentali, mentre nel primo caso il ricorso all'analogia pone sfide concettuali più esigenti.

In questa prospettiva molto generale, comunque, potremmo tentare di schematizzare ${ }^{16} 1^{\prime}$ impatto delle nuove tecnologie sui diritti fondamentali attraverso la seguente classificazione.

A) Le nuove tecnologie hanno in primo luogo creato nuove forme e nuove opportunità per diritti fondamentali già riconosciuti dalle Costituzioni contemporanee.

Gli esempi di nuove opportunità per diritti fondamentali già esistenti sono infiniti. Ha osservato giustamente Paolo Passaglia che

«sulla rete non ci si limita ad esprimersi o a prendere cognizione dell'altrui espressione, ma si fa o si può fare anche molto altro: si studia e si lavora, si fruisce di un servizio pubblico e si adempie ad obblighi, ci si riunisce e ci si associa, si esercita la libertà di iniziativa economica e si esplica la propria libertà sessuale. L'elenco è, come è chiaro, puramente indicativo.... ${ }^{17}$.

La trasformazione imposta alla società dalle nuove tecnologie è stata infatti così pervasiva che un elenco delle nuove opportunità, anche se limitata alle sfere di azione coperte dai diritti fondamentali, sarebbe quasi impossibile da compilare, dato che quasi ogni azione umana compresa fra la nascita e la morte della persona ${ }^{18}$

vol. II, Jovene, Napoli, 2004; A. Lepage, Libertés et droits fondamentaux à l'épreuve de l'internet, Litec, Paris, 2002, nonché il rapporto del Consiglio di Stato francese J. Richard, Les droits fondamentaux à l'épreuve du numérique, Etude annuel du Conseil d'État, Paris, 2014.

${ }_{16}$ Analogamente J. Richard, Les droits fondamentaux, cit., p. 9, il quale osserva che le numerique «renforce la capacité des individus de jouir de certains droits, comme la liberté d'expression ou la liberté d'entreprendre ; il en fragilise d'autres comme le droit à la vie privée, a la sureté et le droit à la securité».

${ }_{17}$ P. Passaglia, Internet nella Costituzione italiana: considerazioni introduttive, in M. Nisticò, P. Passaglia, Internet e Costituzione, Giappichelli, Torino, 2014, p. 15.

1.2. ${ }^{18}$... se non questi stessi due eventi, che in genere non sono oggetto di scelta da parte del diretto interessato. Sul problema della morte digitale si v. G Resta, La "morte" digitale, in Il diritto dell'informazione e dell'informatica, 2014; G. Marino, La «successione digitale», in Osservatorio del Diritto Civile e Commerciale, 2018, n. 1, p. 165-202. A parte sta poi la possibilità stessa di una seconda vita su Internet: si v. E. Bassoli, La disciplina giuridica della seconda vita in Internet: 1'esperienza Second Life, in Informatica e diritto, 2009, n. 1, p. 165 ss. 
ha oggi un modus operandi diverso da quello che aveva prima dell'inizio della rivoluzione informatica e forse anche prima dell' apparizione di internet e dei social media, malgrado che non poche persone siano rimaste estranee alla sfera di azione dei nuovi media (c.d. digital divide).

B) Le nuove tecnologie, tuttavia, consentono anche nuove minacce e ai diritti fondamentali già riconosciuti. Del resto, i due lati della questione sono spesso intrecciati fra loro.

In generale, i mezzi informatici e telematici consentono di acquisire informazioni sulle persone che possono svuotare del tutto la loro privacy ${ }^{19}$. In particolare, le tecniche informatiche permettono di controllare l'intero svolgimento della vita della persona. Le intercettazioni non solo telefoniche, ma anche telematiche, permettono di controllare tutta l'attività individuale che si svolge avendo accesso - nei modi più vari - a qualsiasi computer connesso ad una rete o anche ad un semplice telefono cellulare. Tutte le attività poste in essere da ogni individuo attraverso connessione alla rete sono tracciabili. Più di recente, è diventato possibile innestare da remoto un meccanismo su un telefono cellulare che permette di trasformare tale telefono in un registratore di tutte le attività che la persona svolge: dunque non solo le sue comunicazioni telefoniche e telematiche, ma la sua intera «nuda vita», con una portata totalitaria che avrebbe forse sorpreso anche la fantasia di George Orwell ${ }^{20}$.

Se limitiamo il nostro discorso a Facebook, il più importante dei social media, il suo carattere destrutturato e anarchico pone in crisi tutti i beni giuridici tradizionalmente protetti dai limiti ai diritti fondamentali, alcuni dei quali consistono in beni collettivi - come l'ordine pubblico e la morale - altri in beni individuali (i diritti degli altri) ${ }^{21}$. Da questo secondo punto di vista, una serie di diritti fondamentali, come, in primo luogo, la privacy e l'onore individuale possono essere oggetto di attentati di proporzioni difficilmente immaginabili prima della rivoluzione informatica.

A fronte di questo tipo di minacce si è sviluppato il diritto alla tutela dei dati personali, che è stato disciplinato per lo più a livello europeo ${ }^{22}$, e che nel 2016 è culminato nel Regolamento generale sulla protezione dei dati personali (GDPR),

\footnotetext{
${ }^{19} \mathrm{Su}$ questo problema si v. fra gli altri A. Baldassarre, Il diritto di privacy e la comunicazione elettronica, in Percorsi Costituzionali, 2010, n. 1, p. 49 ss.; F. Pizzetti, La tutela della riservatezza nella società contemporanea, in Percorsi Costituzionali, 2010, n. 1, p. 61 ss.

${ }^{20}$ Si può inoltre ricordare, su un piano diverso, la possibilità di svuotamento del diritto alla proprietà intellettuale.

${ }^{21} \mathrm{Si}$ richiama qui, per il suo valore a nostro avviso paradigmatico, la triade di limiti al diritto al libero sviluppo della personalità prevista nell'art. $2,1^{\circ} \mathrm{comma}$, della Legge fondamentale di Bonn.

${ }^{22}$ Sul diverso approccio a questo problema negli Stati Uniti e nell'Unione europea si v. G. Resta, Governare l'innovazione, cit., p. 204.
} 
entrato in vigore nel 2018, che prevede una serie di principi che dovrebbero minimizzare le minacce alla vita privata della persona ${ }^{23}$.

C) La forma più rilevante di impatto delle nuove tecnologie sui diritti fondamentali è tuttavia rappresentata dall'emersione di nuovi diritti fondamentali ${ }^{24}$, che in alcuni casi (c1) servono a proteggere la persona dalle minacce derivanti dalle nuove tecnologie, mentre in altri (c2) sono finalizzate a rendere effettivamente fruibili le nuove opportunità che le nuove tecnologie hanno generato rispetto ai diritti fondamentali già esistenti.

In Italia, l'emersione di nuovi diritti fondamentali non è sinora avvenuta mediante riforme costituzionali, simili a quelle che sono state invece adottate in altri Paesi (si pensi alla riforma costituzionale messicana del $2005^{25}$ ). Né si può dire che la giurisprudenza costituzionale abbia svolto un ruolo particolarmente attivo in questo campo ${ }^{26}$. Piuttosto, le principali innovazioni hanno avuto origine in ambito europeo, sia mediante l'adozione di atti normativi dell'Unione europea (come le direttive sulla tutela dei dati personali negli anni novanta e nel 2016 il Regolamento Generale citato sopra), sia mediante pronunce della Corte di giustizia dell'Unione europea ${ }^{27}$, mentre un ruolo non meno importante è stato svolto dalla

${ }^{23}$ La dottrina italiana ha dedicato grande attenzione al nuovo Regolamento. Si v. i commenti generali: L. Bolognini, C. Bistolfi, E. Pelino (a cura di), Il regolamento privacy europeo : commentario alla nuova disciplina sulla protezione dei dati personali, Giuffrè, Milano, 2016; G. Finocchiaro (a cura di), Il nuovo regolamento europeo sulla privacy e sulla protezione dei dati personali, Zanichelli, Bologna, 2017; L. Califano, C. Colapietro (a cura di), Innovazione tecnologica e valore della persona - Il diritto alla protezione dei dati personali nel regolamento UE 2016/679, Editoriale Scientifica, Napoli, 2017; G.M. Riccio, G. Scorza, E. Belisario (a cura di), GDPR e normativa privacy: commentario al regolamento (UE) 2016/679 del 27 aprile 2016, Wolters Kluwer, Milano, 2018.

${ }^{24} \mathrm{Si}$ parla qui di «nuovi» diritti in due diversi sensi: si tratta di diritti nuovi in quanto non previsti dalla Costituzione; si tratta altresì di diritti nuovi in quanto emersi in reazione alle nuove tecnologie.

${ }^{25}$ Si pensi inoltre all'art. 5, alinea 72, della Cost. del Brasile del 1988: «è concessa l'azione della habeas data: a) per assicurare la conoscenza di informazioni relative alla persona del richiedente, risultanti da registri o banche dati di enti governativi o di carattere pubblico; $b$ ) per la rettifica di dati, quando non si preferisca farlo tramite procedimento segreto, giudiziario o amministrativo». Sull'habeas data si v. E. Rozo Acuña, Habeas Data costituzionale: nuova garanzia giurisdizionale del diritto pubblico latinoamericano, in Diritto pubblico comparato ed europeo, n. 4, 2002, p. 1923.

${ }^{26} \mathrm{Si} \mathrm{v}$. invece la giurisprudenza costituzionale tedesca, la quale ha elaborato sin dal 1983 (B Verf GE $65,1,43$ ) il diritto all'autodeterminazione informatica (Recht auf informationnelle Selbstbestimmung), ricavandolo dal diritto generale allo sviluppo della personalità (art. 2.1. GG) in combinato disposto con la dignità umana (art. 1.1. GG) e più di recente (BVerfGE 120, 274) il diritto alla garanzia della riservatezza e dell'integrità dei sistemi informativi (Recht auf Gewährleistung der Vertraulichkeit und Integrität informationstechnischer Systeme): cf. J. Ipsen, Staatsrecht II - Grundrechte, XIII ed., Vahlen, München, 2010, p. 82 ss.

${ }^{27} \mathrm{Si}$ v. ad es. le sentenze quelle adottate nei casi Digital rights Ireland (8.4.2014), Google Spain (13.5.2014) e Maximillian Schrems v Data Protection Commissioner, Judgement of the Court (Grand Chamber) 6.10.2015, on Request for a preliminary ruling from the High Court (Ireland). 
legislazione nazionale. Ma non si può trascurare un'altra fonte di novità, sia pure inidonea a produrre immediatamente effetti giuridici: la riflessione dottrinale. Quest'ultima si è sviluppata in maniera molto articolata, cercando di accompagnare le principali novità tecnologiche e giuridiche, leggendole alla luce della tradizione costituzionale (e più in generale della tradizione giuridica) italiana.

La dottrina, in particolare, si è divisa fra coloro che hanno segnalato l'emersione di diversi e specifici nuovi diritti fondamentali (di libertà, sociali, di partecipazione) e coloro che hanno tentato di costruire - sulla base dell'interpretazione dei principi costituzionali italiani - un «macro-diritto fondamentale» (come la c.d. «libertà informatica ${ }^{28}$ ), idoneo ad abbracciare le specifiche situazioni soggettive ritenute meritevoli di tutela. In questa articolazione delle posizioni dottrinali si può vedere la riapparizione di una vecchia alternativa - ben evidente nella dottrina meno recente - nell'interpretazione dei diritti di libertà: una alternativa fra un diritto generale di libertà della persona, poi articolato in una serie di singole facoltà, e una pluralità di diritti di libertà, ciascuno corrispondente alle disposizioni della Costituzione che prevedono tali specifici diritti ${ }^{29}$.

Possiamo forse distinguere i nuovi diritti fondamentali emersi a fronte della società digitale in due categorie.

c1) Anzitutto occorre citare quei nuovi diritti che proteggono la persona da minacce provenienti dall'uso delle nuove tecnologie da parte di soggetti pubblici $\mathrm{o}$, più spesso, privati e che operano seguendo la logica delle libertà o dei c.d. Abwehrrechte:

- il diritto alla protezione dei dati personali, il primo ed il più importante di questi diritti, che si è sviluppato come una costola del diritto alla privacy e che si articola ormai in una serie di facoltà ben distinte fra loro, ciascuna delle quali è talora qualificata come un diritto autonomo, come si può vedere nel General Data Protection Regulation, entrato in vigore nel 2018;

- il diritto all'oblio, o diritto alla privacy storica, una particolare forma di protezione dei dati personali, che ha acquisito una sua autonomia concettuale;

- il «diritto a non essere oggetto di decisioni automatizzate» ${ }^{30} \mathrm{o}$ «diritto fondamentale a non essere "colpiti" da decisioni "esclusivamente" algoritmiche» ${ }^{31}$, che sta emergendo di recente di fronte all'uso degli algoritmi come base di decisioni amministrative pregiudizievoli.

\footnotetext{
${ }^{28} \mathrm{~V}$. infra par. 5.

${ }^{29}$ Mentre sotto la vigenza dello Statuto albertino la prima tendenza era prevalente, dopo l'entrata in vigore della Costituzione repubblicana la dottrina ha ritenuto che esistessero diversi e distinti diritti di libertà costituzionalmente garantiti. Per questa alternativa si v. P.F. Grossi, I diritti di libertà ad uso di lezioni, Giappichelli, Torino, 1991, p. 167 ss.

${ }^{30}$ G. Resta, Governare l'innovazione tecnologica: decisioni algoritmiche, diritti digitali e principio di uguaglianza, in Politica del diritto, 2019, n. 2, p. 222.

${ }^{31}$ A. Simoncini, Profili costituzionali della amministrazione algoritmica, cit., p. 1175.
} 
c2) Si è però anche dato il caso di un nuovo diritto fondamentale di diversa natura, comparabile ai diritti sociali o a prestazione, in quanto volto a permettere alla generalità dei cittadini di fruire di un mezzo di comunicazione sempre più imprescindibile per l'esercizio di molti diritti fondamentali: è il caso del diritto di accesso ad Internet, che ha trovato riconoscimento a livello costituzionale nell' art.3, comma 6, della Costituzione messicana e del quale si è molto discusso in vari ordinamenti, fra cui l'Italia, per ora attorno ad innovazioni legislative che hanno tentato di far fronte al c.d. digital divide ${ }^{32}$.

D) A fronte delle nuove minacce generate dalla nuove tecnologie, in Italia come in altri Stati sono state introdotte norme legislative finalizzate a proteggere alcuni interessi umani fondamentali, che hanno generato nuovi limiti e nuove restrizioni di alcuni diritti fondamentali già riconosciuti, anzitutto la libertà di espressione, enucleando comportamenti vietati, talora mediante norma penale. Questa forma di impatto è diversa da quella indicata sub b) in quanto in quel caso si ha una innovazione proveniente dai fatti (le nuove tecnologie e le possibilità da esse aperte), mentre in questo si tratta di nuovi limiti e restrizioni previsti da regole giuridiche per bilanciare effetti nocivi dell'uso delle tecnologie.

In Italia questo è il caso del reato di c.d. «revenge porn» (diffusione illecita di immagini o video sessualmente espliciti senza il consenso delle persone rappresentate), introdotto dalla legge n. 69/2019, che protegge beni giuridici già tutelati, come la privacy e la dignità della persona, ma lo fa vietando comportamenti in precedenza non proibiti e forse riconducibili a forme - seppur anomale - di esercizio della libertà di espressione da parte dell'autore dell'atto.

Un altro esempio può forse essere visto nelle norme volte a reprimere il possesso e lo scambio online di materiale pedo-pornografico.

Manca per ora in Italia una legislazione volta specificamente a contrastare l'odio in rete, del tipo di quella recentemente approvata dall'Assemblea nazionale francese e modificata dal Senato francese nel dicembre 2019.

\section{$3 \quad$ Le tecniche di contemperamento fra $\mathrm{i}$ diritti}

Ovviamente in questo contesto le consolidate tecniche di bilanciamento, proporzionalità e ragionevolezza svolgono un ruolo importante per contemperare i diritti e i loro limiti. Infatti, come si è già accennato, i diritti fondamentali - vecchi

\footnotetext{
${ }^{32}$ Sulle diverse dimensioni del digital divide, si v. P. Passaglia, Internet e Costituzione, cit., p. 18. il quale elenca cinque diverse forme di esclusione da Internet: a) la determinazione autoritativa, correlata a determinate situazioni (ad es. lo stato di detenzione); b) le carenze infrastrutturali,che rendono impossibile la connessione in determinate aree; c) le difficoltà economiche che impediscono a un individuo di possedere o detenere strumenti che consentano un adeguato accesso alla rete; d) problemi di ordine fisico che precludono al soggetto la fruizione di determinati contenuti; e) un deficit culturale dell'individuo, privo delle conoscenze informatiche sufficienti per interfacciarsi con un computer.
} 
e nuovi - la cui tutela si impone di fronte alle nuove tecnologie interagiscono sia con altri diritti, sia con principi costituzionali di notevole pregio.

Un esempio di conflitti fra diritti o fra diritti e principi in questo contesto si può vedere in un caso recentemente deciso dalla Corte costituzionale italiana con la sent. n. 20/2019.

La Corte si trovava a giudicare su alcune norme legislative che imponevano ai dirigenti amministrativi, in nome del principio di trasparenza della pubblica amministrazione e della lotta contro la corruzione, una serie di incisivi obblighi di pubblicazione on-line di informazioni relative alla loro persona ${ }^{33}$.

Fra i dati di cui era richiesta la pubblicazione vi erano «i compensi di qualsiasi natura connessi all'assunzione della carica, gli importi di viaggi di servizio e missioni pagati con fondi pubblici (...); la dichiarazione dei redditi soggetti all'imposta sui redditi delle persone fisiche e quella concernente i diritti reali su beni immobili e su beni mobili iscritti in pubblici registri, le azioni di società, le quote di partecipazione a società, anche in relazione al coniuge non separato ed ai parenti entro il secondo grado, ove essi vi acconsentano, dovendosi in ogni caso dare evidenza al mancato consenso (...); l'ammontare complessivo degli emolumenti percepiti da ciascun dirigente a carico della finanza pubblica».

In virtù di tali obblighi, osserva la sent. n. 20/2019, «la totalità della dirigenza amministrativa è stata sottratta al regime di pubblicità congegnato dall'art. 15 del d.lgs. n. 33 del 2013 - che per essi prevedeva la pubblicazione dei soli compensi percepiti, comunque denominati - ed è stata attratta nell'orbita dei ben più pregnanti doveri di trasparenza originariamente riferiti ai soli titolari di incarichi di natura politica» (punto 5.1).

Alcuni dei funzionari interessati da tali obblighi, li contestavano in nome del diritto alla privacy. La Corte costituzionale italiana ha quindi avuto modo di pronunciarsi in forma generale proprio sul delicato nodo del rapporto fra privacy e trasparenza.

«I diritti alla riservatezza e alla trasparenza si fronteggiano soprattutto nel nuovo scenario digitale: un ambito nel quale, da un

\footnotetext{
${ }^{33}$ Oggetto della questione di legittimità costituzionale era l'art. 14, commi 1-bis e 1-ter, del decreto legislativo 14 marzo 2013, n. 33 (Riordino della disciplina riguardante il diritto di accesso civico e gli obblighi di pubblicità, trasparenza e diffusione di informazioni da parte delle pubbliche amministrazioni). Tali commi erano stati inseriti nell'art. 14 del d.lgs. n. 33 del 2013 dall'art. 13, comma 1, lettera c), del decreto legislativo 25 maggio 2016, n. 97 (Revisione e semplificazione delle disposizioni in materia di prevenzione della corruzione, pubblicità e trasparenza, correttivo della legge 6 novembre 2012, n. 190 e del decreto legislativo 14 marzo 2013, n. 33, ai sensi dell'articolo 7 della legge 7 agosto 2015, n. 124, in materia di riorganizzazione delle amministrazioni pubbliche). In tal modo erano state estese ai titolari di incarichi dirigenziali nella pubblica amministrazione (quale che fosse il titolo cui erano stati conferiti), gli obblighi di pubblicazione di una serie di dati, che in precedenza erano previsti dallo stesso art. 14, comma 1, del d.lgs. n. 33 del 2013 per i titolari di incarichi politici.
} 
lato, i diritti personali possono essere posti in pericolo dalla indiscriminata circolazione delle informazioni, e, dall'altro, proprio la più ampia circolazione dei dati può meglio consentire $a$ ciascuno di informarsi e comunicare (punto 2.2). (...)

Si è in presenza di una questione concernente il bilanciamento tra due diritti: quello alla riservatezza dei dati personali, inteso come diritto a controllare la circolazione delle informazioni riferite alla propria persona, e quello dei cittadini al libero accesso ai dati ed alle informazioni detenuti dalle pubbliche amministrazioni.

In valutazioni di tale natura, il giudizio di ragionevolezza sulle scelte legislative si avvale del cosiddetto test di proporzionalità, che «richiede di valutare se la norma oggetto di scrutinio, con la misura e le modalità di applicazione stabilite, sia necessaria e idonea al conseguimento di obiettivi legittimamente perseguiti, in quanto, tra più misure appropriate, prescriva quella meno restrittiva dei diritti a confronto e stabilisca oneri non sproporzionati rispetto al perseguimento di detti obiettivi».

La Corte ha inoltre sottolineato che, nell'operare il bilanciamento fra diritto alla riservatezza e obblighi di pubblicazione (con conseguente possibile trattamento) di dati personali imposti dal principio di trasparenza si debbano seguire $\mathrm{i}$ «principi di proporzionalità, pertinenza e non eccedenza, in virtù dei quali deroghe e limitazioni alla tutela della riservatezza di quei dati devono operare nei limiti dello stretto necessario».

Sulla base di tali criteri, la Corte costituzionale ha ritenuto che l'obbligo per i dirigenti amministrativi di pubblicare tutte le «dichiarazioni e attestazioni contenenti dati reddituali e patrimoniali (propri e dei più stretti congiunti), ulteriori rispetto alle retribuzioni e ai compensi connessi alla prestazione dirigenziale» non fosse giustificato e fosse pertanto costituzionalmente illegittimo, trattandosi «di dati che non necessariamente risultano in diretta connessione con l'espletamento dell'incarico affidato».

La Corte costituzionale ha ritenuto che alcuni obblighi generalizzati di pubblicazione non rispondessero «alle due condizioni richieste dal test di proporzionalità: l'imposizione di oneri non sproporzionati rispetto ai fini perseguiti, e la scelta della misura meno restrittiva dei diritti che si fronteggiano», violando pertanto «l'art. 3 Cost., innanzitutto sotto il profilo della ragionevolezza intrinseca. (...) L'onere di pubblicazione in questione risulta, in primo luogo, sproporzionato rispetto alla finalità principale perseguita, quella di contrasto alla corruzione nell'ambito della pubblica amministrazione». La Corte ha pertanto ritenuto che l'obbligo generalizzato di pubblicazione fosse in contrasto anche con il principio per cui, "nelle operazioni di bilanciamento, non può esservi un decremento di tutela di un diritto fondamentale se ad esso non fa riscontro un corrispondente incremento di tutela di altro interesse di pari rango". Inoltre «anche 
sotto il ... profilo ... della necessaria scelta della misura meno restrittiva dei diritti fondamentali in potenziale tensione, la disposizione censurata non supera il test di proporzionalità».

$\mathrm{Al}$ di là delle peculiarità di questo caso, si può vedere come ciò che è decisivo, come molto spesso accade in questioni relative all'interpretazione dei diritti fondamentali, non è solo il rango costituzionale degli interessi coinvolti, quanto il modo in cui essi sono bilanciati e in cui i limiti sono proporzionati ai diritti.

\section{Alcuni nodi del dibattito costituzionalistico italiano a proposito di Internet}

Fatte queste premesse di carattere metodologico, occorre ora mettere in evidenza alcuni nodi del dibattito costituzionalistico italiano che potrebbero avere un certo interesse anche per un pubblico brasiliano.

Mi limiterò qui ai profili costituzionalistici, tralasciando molti altri ambiti di discussione, che hanno investito tutte le discipline giuridiche, dal diritto civile (ad es. la responsabilità degli Internet service providers, ${ }^{34}$ la tutela della personalità digitale $^{35}$, ecc.) al diritto penale (ad es. sul reato di diffamazione via web e in particolare via social media o al già citato reato di revenge porn), dalla procedura penale (si v. l'intercettazione telefonica mediante trojan, introdotto dalla c.d. legge Spazzacorrotti) al diritto amministrativo (con gli sforzi per lo sviluppo di una amministrazione digitale), dal diritto internazionale (si v. gli studi sui Big Data) al diritto commerciale (il commercio elettronico), eccetera. Non voglio però tacere su un dato: in una dottrina giuridica molto compartimentalizzata in diversi settori scientifico-disciplinari poco comunicanti fra loro, come quella italiana, in cui gli studiosi dei diversi settori del diritto studiano temi simili gomito a gomito, ma quasi in maniera parallela, con scarsi incontri fra le rispettive discipline, questo tema, se non altro per la sua novità, ha prodotto felici interazioni tra studiosi di discipline giuridiche diverse e anche un buon grado di apertura ai risultati di scienze esatte (informatica) o di scienze sociali (sociologia e scienza politica, economia) che hanno dato decisivi contributi su questi temi.

Su questi temi operano da anni alcune importanti riviste ${ }^{36}$ e sono stati prodotti significativi lavori monografici, alcuni dei quali con «taglio» costituzionalistico.

\section{La libertà informática}

Già negli anni novanta del XX secolo, Vittorio Frosini aveva proposto di utilizzare il concetto di libertà informatica per ricondurre alla logica dei diritti fondamentali i problemi posti dai computer e poi da Internet. Nella sua originaria

${ }^{34} \mathrm{~V}$. ad es. R. Panetta, Il ruolo dell'Internet service provider e $i$ profili di responsabilità civile, in Responsabilità Civile e Previdenza, 2019, n. 3, p. 1017 e ss.

${ }^{35} \mathrm{Su}$ cui v. ad es. S. Landini, Identità digitale tra tutela della persona e proprietà intellettuale, in Rivista di Diritto Industriale, 2017, n. 4-5. p. 180 e ss.

${ }^{36}$ Le principali sono Il diritto dell'informazione e dell'informatica e Informatica e diritto. 
versione, quella esposta nel 1981, la libertà informatica veniva raffigurata come positiva e negativa. La libertà informatica negativa esprime

«il diritto di non rendere di dominio pubblico certe informazioni di carattere personale, privato, riservato (qualifiche queste, che potrebbero in certi casi non coincidere tra loro); la libertà informatica positiva, invece, esprime la facoltà di esercitare un diritto di controllo sui dati concernenti la propria persona che sono fuoriusciti dalla cerchia della privacy per essere divenuti elementi di input di un programma elettronico.

Questa seconda dimensione ha acquisito maggiore importanza con l'apparizione e la diffusione di Internet: il diritto di libertà informatica

«è diventato una pretesa di libertà in senso attivo, non libertà $d a$ ma libertà $d i$, che è quella di valersi degli strumenti informatici per fornire e ottenere informazioni di ogni genere. $E^{\prime}$ il diritto di partecipazione alla società virtuale, che è stata generata dall'avvento degli elaboratori elettronici nella società tecnologica: è una società dai componenti mobili e dalle relazioni dinamiche, in cui ogni individuo partecipante è sovrano nelle sue decisioni» ${ }^{37}$.

Oggi il concetto di libertà informatica è riproposto da alcuni studiosi, fra cui Tommaso Edoardo Frosini, figlio di Vittorio, ma non è accolto in generale dalla dottrina ${ }^{38}$, né è stato recepito nella giurisprudenza costituzionale.

La nozione di libertà informatica ha la pretesa di abbracciare in un unico e nuovo diritto fondamentale la tutela costituzionale della persona di fronte ai mezzi informatici - quasi un «Computergrundrecht», per riprendere una terminologia di origine tedesca - e in particolare di fronte a Internet.

Una analoga pretesa di costruire un nuovo diritto, idoneo a tutelare in via generale le persone dalle conseguenze della rivoluzione telematica si può ritrovare nella ricostruzione proposta da Paolo Passaglia, il quale ha sostenuto che la tutela della persona di fronte a Internet deve muovere dalla constatazione della globalità dell'impatto di questo mezzo sulla vita individuale e collettiva, con la conseguenza che Internet non può essere ridotto ad un mezzo di comunicazione o di diffusione del pensiero (rilevante, rispettivamente, alla luce dell'art. 15 o dell'art. 21 della Costituzione italiana), ma deve essere collegato allo sviluppo della personalità individuale, di cui Internet costituisce ormai uno strumento indispensabile. Per

${ }^{37}$ V. Frosini, L'orizzonte giuridico dell'Internet, in Il dritto dell'Informazione e dell'informatica, 2000, p. 275.

${ }^{38} \mathrm{Si}$ v. ad es. le persuasive critiche di M. Betzu, Interpretazione e sovra-interpretazione dei diritti costituzionali nel cyber-spazio, p. 460 al suo fondamento costituzionale. 
questo motivo, la tutela costituzionale dovrebbe essere duplice: da un lato, nella prospettiva dell'art. 2 della Cost. italiana, si tratterebbe, appunto di una forma di libero sviluppo o svolgimento della personalità cui la Costituzione offre tutela; dall'altro, la constatazione dell'esistenza di vari tipi di ostacoli - il c.d. digital divide - al pieno sviluppo della personalità individuale mediante l'accesso ad Internet chiamerebbe in causa il c.d. principio di eguaglianza sostanziale (art. 3, comma 2, Cost.), vale a dire l'obbligo per la Repubblica, nelle sue diverse dimensioni, di operare per «rimuovere gli ostacoli» che impediscono tale sviluppo:

«Il diritto di libertà (ma non solo ...) consistente nello svolgimento della propria personalità nel mondo virtuale non può prescindere da un generalizzato diritto a prestazione che chiama i pubblici poteri ad un intervento costante. Ecco quindi che il "riconoscere" ed il "garantire" di cui all'art. 2 della Costituzione si traducono, alla luce dell'art. 3, comma 2, non già in una presa d'atto ed in una protezione ex post dei diritti, ma postulano un'azione positiva di creazione delle condizioni perché il riconoscimento sia effettivo e la garanzia sia il presupposto per l'esercizio dei diritti e non solo la risposta al loro impedimento» ${ }^{39}$.

\section{L'accesso a Internet come diritto fondamentale.}

L'accesso a Internet in condizioni non discriminatorie e dietro corrispettivo monetario è riconosciuto nell'ordinamento italiano dall'art. 54 del Decreto legislativo n. 259/2003, il c.d. Codice delle comunicazioni elettroniche, che recepisce in Italia la direttiva 2002/19/CE del Parlamento e del Consiglio del 7.3.2002. Si tratta dunque di una disciplina di rango legislativo. Una proposta di introdurre una disposizione costituzionale che riconosca esplicitamente il diritto di accesso ad Internet è stata per ora discussa ma non approvata dal Parlamento italiano.

Una parte della dottrina italiana ritiene che già oggi si possa un riconoscere fondamento costituzionale implicito a tale diritto nell'art. 2 della Costituzione, che in Italia svolge fra l'altro la funzione di apertura del catalogo costituzionale ai c.d. «nuovi diritti». In questo senso si è espresso con nettezza Tommaso Edoardo Frosini, che ha fra l'altro collegato questo diritto alla concezione della libertà informatica elaborata da Vittorio Frosini:

«Il diritto di accesso a Internet è da considerarsi un diritto sociale, o meglio una pretesa soggettiva a prestazioni pubbliche, al pari dell'istruzione, della sanità e della previdenza. Un servizio universale, che le istituzioni nazionali devono garantire ai loro cittadini attraverso investimenti statali, politiche sociali ed educative, scelte di spesa pubblica. Infatti: sempre di più l'accesso

${ }^{39}$ P. Passaglia, Internet e Costituzione, p. 21 
alla rete Internet, e lo svolgimento su di essa di attività, costituisce il modo con il quale il soggetto si relaziona con i pubblici poteri, e quindi esercita i suoi diritti di cittadinanza ${ }^{40}{ }^{4}$.

La collocazione sistematica di questo nuovo diritto fondamentale è stata evidenziata osservando che

«il diritto di accesso a Internet è una libertà fondamentale il cui esercizio è strumentale all'esercizio di altri diritti e libertà costituzionali: non solo la libertà di manifestazione del pensiero di cui all'art. 21 Cost., ma anche il diritto al "pieno sviluppo della persona umana" e "all'effettiva partecipazione di tutti i lavoratori all'organizzazione politica, economica e sociale del Paese" di cui all'art. 3 Cost., o piuttosto la libertà di impresa di cui all'art. 41 Cost.» ${ }^{41}$

Ne deriverebbe una serie di conseguenze in termini di compiti per i poteri pubblici:

«questo nuovo modo di intendere il rapporto cittadino e amministrazione in termini di una nuova cittadinanza digitale, deve trovare una sua piena alfabetizzazione informatica, quale diritto sociale di cui la Repubblica deve assicurare a tutti la fruizione, e in particolare il diritto all'istruzione e il diritto allo sviluppo culturale informatico, ritenuto dalla Corte costituzionale - sent. 307/2004 - "corrispondente a finalità di interesse generale, quale è lo sviluppo della cultura, nella specie attraverso l'uso dello strumento informatico, il cui perseguimento fa capo alla Repubblica in tutte le sue articolazioni (art. 9 Cost.) $)^{\prime \prime}{ }^{42}$.

«L'accesso a Internet è diventato, per milioni di cittadini, parte integrante e condizione di esercizio di numerosi diritti e libertà di valore costituzionale; inibire l'accesso, allora, vorrebbe essere una

${ }^{40}$ T.E. Frosini, Il diritto costituzionale di accesso a Internet, in Rivista AIC, n. 1/2011, p. 8. Su questo tema v. altresì P. Passaglia, L'accesso a Internet è un diritto (il Conseil Constitutionnel francese dichiara l'incostituzionalità di parte della c.d. "legge anti file-sharing"), in Il Foro Italiano, 2009, IV, p. 473 ss.; R. Pisa, L'accesso a Internet: un nuovo diritto fondamentale? wwww.treccani.it/Portale/sito/diritto/approfondimenti/2 Pisa internet.html; L. Marino, Le droit d'accès à internet, nouveau droit fondamental, in Recueil Dalloz, n. 30, 2009, p. 2045 ss. ; F. Badocco, Riflessioni sul diritto d'accesso a Internet nell'ambito del diritto dell'Unione europea, in Informatica e diritto, 2009, $\mathrm{n}$. 1, p. 153 ss.

${ }^{41}$ T.E. Frosini, Il diritto costituzionale di accesso a Internet, p. 9-10.

${ }^{42}$ T.E. Frosini, Il diritto costituzionale di accesso a Internet, p. 8-9. 
sanzione sproporzionata nella misura in cui avrebbe un'incidenza grave e diretta sull'esercizio di tali diritti. E quindi, prevedere e imporre la disconnessione da Internet senza la previa autorizzazione dell'autorità giudiziaria confligge con l'art. 11 della Dichiarazione del 1789 e costituisce, pertanto, una violazione sproporzionata della libertà di espressione ${ }^{43}{ }^{3}$.

Ma una parte della dottrina ha criticato questa ricostruzione, considerandola come un esempio di «sovra-interpretazione» delle disposizioni costituzionali ed è giunta alla conclusione che un diritto di questo tipo non trova riconoscimento nel sistema costituzionale vigente. Esso potrebbe avere tuttavia rilievo muovendo dalla qualificazione di Internet quale mezzo (e in taluni casi presupposto ormai necessario) per il godimento di diritti sociali costituzionalmente garantiti, ad es. in materia di salute, lavoro e istruzione ${ }^{44} \mathrm{o}$ in correlazione con i diritti di libertà di comunicazione e di manifestazione del pensiero ${ }^{45}$.

Altri invece ha invocato a sostegno del riconoscimento del diritto di accesso ad Internet come di diritto positivo vigente una sentenza nella quale la Corte costituzionale ha giustificato una legge statale che aveva istituito fondi speciali finalizzati a incentivare l'acquisto di personal computer da parte di giovani e di altri soggetti a reddito ridotto come non invasiva delle competenze legislative regionali, ma come corrispondente

«a finalità di interesse generale, quale è lo sviluppo della cultura, nella specie attraverso l'uso dello strumento informatico, il cui perseguimento fa capo alla Repubblica in tutte le sue articolazioni (articolo 9 della Costituzione), anche al di là del riparto di competenze per materia fra Stato e Regioni di cui all'art. 117 della Costituzione» ${ }^{46}$.

\section{Alcune questioni in materia di comunicazione e di espressione}

In questo complesso panorama, però, alcuni specifici diritti di libertà «classici» hanno importanza centrale: si tratta da un lato della libertà di corrispondenza e di

\footnotetext{
${ }^{43}$ T.E. Frosini, Il diritto costituzionale di accesso a Internet, p.13. Frosini usa poi argomenti di diritto comparato o derivati dall'evoluzione della legislazione ordinaria. Cita la sentenza n. 2009-580 DC del 10 giugno 2009 del Consiglio costituzionale francese e la sentenza della Sala Constitucional del Costarica n. 12790 del 30.7.2010. Ricorda infine che la «legge n. 4/2004 ("legge Stanca"), relativa alle disposizioni per favorire l'accesso dei soggetti disabili agli strumenti informatici ... la quale riconosce e tutela il diritto di ogni persona ad accedere a tutte le fonti di informazione e ai relativi servizi, compresi quelli che si articolano attraverso gli strumenti informatici e telematici».

${ }^{44}$ Così M. Betzu, Interpretazione e sovra-interpretazione dei diritti costituzionali nel cyber-spazio, p. 463.

${ }^{45} \mathrm{M}$. Betzu, Regolare Internet, cit., p. 89.

${ }^{46}$ Sent. n. 307/2009 della Corte costituzionale, n. 3.1. del cons. in diritto.
} 
comunicazione ${ }^{47}$ e dall'altro della libertà di espressione o di manifestazione del pensiero $^{48}$. Dato che in Internet si sono sviluppate nuove forme di comunicazione (e-mail, mailing list, chat, social networks, newsgroups, forum, blog, siti Internet, per non citare che le sole forme più note ai profani), diverse da quelle esistenti prima della sua creazione, è evidente che la sfera di azione di questi due diritti fondamentali (che potremmo anche chiamare Kommunikationsgrundrechte) è stata immediatamente chiamata in causa dallo sviluppo della Rete. Le libertà comunicative - che devono essere distinte in quanto la comunicazione è rivolta a destinatari determinati (e potenzialmente privati) nella libertà di comunicazione ${ }^{49}$, mentre è rivolta a destinatari indeterminati nella manifestazione del pensiero sono senza dubbio quelle il cui senso è stato più profondamente modificato dall'avvento di Internet.

Il tema della libertà di espressione via Internet è stato analizzato con attenzione dalla dottrina italiana, avendo riguardo sia alle nuove forme esercizio di questo diritto - tradizionalmente molto studiato nell'Italia del dopoguerra, anche se lo studio di esso si è concentrato soprattutto sui «mezzi di diffusione del pensiero» (stampa e televisione) ${ }^{50}$ - sia al ripensamento dei limiti ad esso costituzionalmente opponibili nel contesto generale di Internet ${ }^{51}$ e in particolare, più di recente, in quello dei social media.

Riguardo alle forme di espressione, si può citare il dibattito sull'esistenza di un diritto all'anonimato, vale a dire sulla possibilità di ricavare dalla libertà di manifestazione del pensiero prevista dalla Costituzione italiana un diritto all'espressione di pensieri in forma anonima, qualora colui che esprime il pensiero intenda sottrarsi all'identificazione. Com'è noto, Internet amplia notevolmente le possibilità pratiche di esercizio di questo diritto in forma anonima. Al riguardo, due opinioni si sono confrontate. Secondo la prima, che riprende orientamenti

${ }^{47}$ Art. 15 della Costituzione italiana e art. 8 della Convenzione europea dei diritti dell'uomo.

${ }^{48}$ Art. 21 della Costituzione italiana e art. 10 della Convenzione europea dei diritti dell'uomo.

${ }^{49}$ Pertanto la libertà di corrispondenza garantisce i fenomeni della e-mail e della chat, mentre per i fenomeni della mailing list e del social network il confine fra questa libertà e quella di espressione è più delicato, e dipende dal numero dei soggetti coinvolti nella conversazione.

50 «Il significato di questa libertà è stato condizionato, se non addirittura identificato, in modo decisivo dal mezzo attraverso il quale si esercita il diritto di manifestare liberamente il pensiero» (TE Frosini, Il diritto costituzionale di accesso a Internet, p. 7).

${ }^{51}$ A. Papa, Espressione e diffusione del pensiero in Internet. Tutela dei diritti e progresso tecnologico, Giappichelli, Torino, 2009; S. Sica e V. Zeno Zencovich, Legislazione, giurisprudenza e dottrina nel diritto dell'Internet, in Il diritto dell'informazione e dell'informatica, 2010, n. 3, p. 377 ss.; M. Viggiano, Internet. Informazione, regole e valori costituzionali, Jovene, Napoli, 2010; V. Zeno Zencovich, Manifestazione del pensiero, libertà di comunicazione e la sentenza sul caso "Internet", in Il diritto dell'informazione e dell'informatica, 1996, n. 4/5,; V. Zeno Zencovich, Perché occorre rifondare il significato della libertà di manifestazione del pensiero, in Percorsi Costituzionali, 2010, n. 1, p.69 ss. Fra i contributi anglosassoni si v. J.M. Balkin, The Future of Free Expression in a Digital Age, in Pepperdine Law Review, 36, 2008, p. 101 ss. 
molto diffusi nella dottrina statunitense, l'anonimato sarebbe giustificato dall'esigenza di allargare le possibilità di espressione del più vario tipo di pensieri, per sviluppare al massimo il libero mercato delle idee. Per la seconda opinione, la libertà di opinione, pur sottratta di norma a restrizioni preventive, sarebbe soggetta a responsabilità successive, che potrebbero essere fatte valere solo ove l'anonimato non sia garantito, o lo sia solo in forma relativa, nel senso che esisterebbe un diritto ad esprimere un pensiero in forma anonima, ma l'esigenza di accertare eventuali responsabilità renderebbe possibile esigere l'individuazione dell'autore del pensiero espresso anonimamente. Il testo della Costituzione italiana consente di ritenere che l'anonimato non sia di per sé garantito in base a due argomenti: a) da un lato l'art. 21 riconosce il diritto a manifestare liberamente il «proprio» pensiero, vale a dire il pensiero dell'autore, inteso come soggetto individuato; $b$ ) dall'altro lo stesso art. 21 consente il sequestro degli stampati per la violazione delle norme sulla individuazione dei responsabili: ne segue che questi devono essere individuati o, quantomeno, individuabili ${ }^{52}$.

Ma le sfide che Internet pone alla libertà di manifestazione del pensiero sono ben più ardue. Ha giustamente osservato Tommaso Edoardo Frosini che «Internet ha consentito il recupero della nozione di manifestazione del pensiero come libertà individuale, cioè senza "filtri", ovvero senza mediazioni di sorta, un open network ${ }^{53}$. Se la libertà di opinione, di espressione e di pensiero aveva finito per passare in secondo piano nella storia costituzionale italiana rispetto alla libertà di stampa e alla libertà della radiotelevisione ed ai problemi del pluralismo (esterno ed interno) dei mezzi di comunicazione, nonché alle questioni relative alla professione di giornalista, Internet ha esaltato la libertà di pensiero del quisque de populo. Eliminati i «filtri» (che in precedenza erano affidati ai media), esso ha posto in maniera assai più radicale che in passato la questione dei limiti alla libertà di espressione e, assieme ad essi, quella della tolleranza.

\section{I social networks e i motori di ricerca come destinatari dei diritti fondamentali}

La possibilità di esercitare automaticamente su Internet i diritti fondamentali, in forme analoghe e con contenuti e limiti analoghi a quelli del mondo off-line dipende tuttavia da diversi fattori. Ovviamente il primo fattore ha natura pratica, e consiste nella possibilità giuridica e materiale di accedere ad Internet, su cui si è ragionato nel par. 6 . Il secondo problema ha natura soggettiva e dipende da tutti

\footnotetext{
52 Per il dibattito italiano sul diritto all'anonimato si v. G.M. Riccio, Diritto all'anonimato $e$ responsabilità civile del provider, in L. Nivarra, V. Ricciuto (a cura di), Internet e il diritto dei privati, Giappichelli, Torino, 2002; P. Costanzo, Profili costituzionali di Internet, in G. Tosi (a cura di), I problemi giuridici di Internet, Giuffrè, Milano, 2003, p. 73 ss.; E. Pelino, L'anonimato su Internet, in G. Finocchiaro, Diritto all'anonimato, Cedam, Padova, 2008; A. Papa, Espressione e diffusione, cit., 86 ss.; M. Betzu, Regolare Internet, cit., p. 142 ss.

${ }^{53}$ T.E. Frosini, Il diritto costituzionale di accesso a Internet, cit., p 7.
} 
quei fattori che - considerati insieme - determinano l'alfabetizzazione digitale della persona.

Ma una volta ottenuto accesso alla rete, l'esercizio dei diritti fondamentali incontra una serie di problemi che riguardano l'azione di una serie di soggetti che sulla rete si configurano come veri e propri poteri privati, talora di dimensioni (tecnologiche ed economiche) gigantesche. Con questi soggetti l'utente entra in contatto sulla base di regole di diritto privato, concludendo con essi un contratto ${ }^{54}$, talora a titolo gratuito, talora a titolo oneroso ${ }^{55}$. Si pone dunque immediatamente la questione - che nella teoria dei diritti fondamentali è concettualizzabile attorno al problema dei «destinatari dei diritti fondamentali» - delle regole applicabili ai rapporti fra l'utente e il soggetto di diritto privato che fornisce servizi su Internet: si tratta di un rapporto regolato dal contratto o dalla Costituzione, e in particolare dai diritti fondamentali in essa previsti?

Un buon esempio ci può essere offerto da Facebook. Questa società commerciale è ormai un mezzo decisivo per l'esercizio dei diritti fondamentali, in quanto essa, ad es.:

1. consente la comunicazione privata fra due o più soggetti, ovvero fra l'emittente di un messaggio e uno o più destinatari determinati.

2. consente la comunicazione potenzialmente pubblica fino al raggiungimento di destinatari indeterminati, dunque la manifestazione del pensiero, sfuggendo alle mediazioni rappresentate da imprese di comunicazione.

3. consente l'organizzazione immediata di riunioni fisigne (c.d. flashmob) ${ }^{56}$, nonché l'organizzazione di riunioni virtuali.

4. consente la formazione di gruppi virtuali, quasi una forma di esercizio virtuale della libertà di associazione.

\footnotetext{
${ }^{54}$ Sulla natura di questi servizi si v. G. Resta, V. Zeno-Zencovich, Volontà e consenso nella fruizione dei servizi in rete, in Rivista Trimestrale di Diritto e Procedura Civile, 2018, n. 2, p. 411-440.

${ }^{55}$ Sulla natura del contratto si v. ora la sentenza del TAR Lazio del 10 gennaio 2020, nella quale il giudice amministrativo ha sostenuto che il servizio reso da Facebook non ha natura gratuita, dato l'uso dei dati personale da parte di tale impresa, sul quale si basa gran parte dei suoi proventi. Di tale sentenza è al momento disponibile solo la notizia di stampa: https://www.repubblica.it/economia/diritti-e-consumi/diritticonsumatori/2020/01/10/news/ facebook non e gratuito il tar conferma una delle due sanzi oni antitrust-245423971/?ref=RHPPLF-BH-I0-C8-P8-S1.8-T1.

${ }^{56}$ Altra cosa, invece, è ritenere che Facebook e più in generale Internet, consentano lo svolgimento di riunioni telematiche che siano qualificabili come riunioni ai sensi dell'art. 17 Cost. anziché come comunicazioni o manifestazioni del pensiero ex art. 15 o 21 Cost., come sostengono A. Pirozzoli, La libertà di riunione in Internet, in Dir. Inf., 2004, p. 623 e M. Villone, Internet e la politica, in M. Villone, A. Ciancio, G. De Minico, G. Demuro, F. Donati, Nuovi mezzi di comunicazione e identità. Omologazione o diversità?, Aracne, Roma, 2012, p. 565. Per la critica di questa tesi si v. M. Betzu, Interpretazione e sovra-interpretazione dei diritti costituzionali nel cyber-spazio, in M. Villone, A. Ciancio, G. De Minico, G. Demuro, F. Donati, Nuovi mezzi di comunicazione, cit., p. 459.
} 
5. consente l'organizzazione e l'interazione stabile di gruppi di carattere politico.

6. consente tutte le attività comunicative ai soggetti collettivi del più vario tipo (fra cui ad es. quelli di carattere religioso).

7. consente le attività comunicative di soggetti istituzionali, nonché di attori politici del più vario tipo.

La questione che si pone è allora se Facebook sia giuridicamente legittimato a delimitare tali attività in base a principi di carattere ideologico, cui scelga di ispirarsi. Un recente caso verificatosi in Italia consente qualche primo ragionamento sul punto ${ }^{57}$. Il 9 settembre 2018 Facebook ha oscurato una serie di profili di esponenti politici di estrema destra e in particolare il profilo Facebook dell'organizzazione Casapound, di orientamento ideologico neofascista. I rappresentanti legali di tale organizzazione hanno presentato ricorso di urgenza al Tribunale di Roma ai sensi dell'art. 700 del codice di procedura civile e il giudice monocratico di tale Tribunale ha adottato un provvedimento cautelare nel quale ha ordinato a Facebook di riattivare tali profili. E' interessante la motivazione di questo provvedimento (contro il quale Facebook ha presentato ricorso):

$\ll E^{\prime} \quad$.. evidente il rilievo preminente assunto dal servizio di Facebook (o di altri social network ad esso collegati) con riferimento all'attuazione di principi cardine essenziali dell'ordinamento come quello del pluralismo dei partiti politici (49 Cost.), al punto che il soggetto che non è presente su Facebook è di fatto escluso (o fortemente limitato) dal dibattito politico italiano, come testimoniato dal fatto che la quasi totalità degli esponenti politici italiani quotidianamente affida alla propria pagina Facebook i messaggi politici e la diffusione delle idee del proprio movimento. Ne deriva che il rapporto tra Facebook e l'utente che intenda registrarsi al servizio (o con l'utente già abilitato al servizio come nel caso in esame) non è assimilabile al rapporto tra due soggetti privati qualsiasi in quanto una delle parti, appunto Facebook, ricopre una speciale posizione: tale speciale posizione comporta che Facebook, nella contrattazione con gli utenti, debba strettamente attenersi al rispetto dei principi costituzionali e ordinamentali finchè non si dimostri (con accertamento da compiere attraverso una fase a cognizione piena) la loro violazione da parte dell'utente. Il rispetto dei principi costituzionali e ordinamentali costituisce per il soggetto Facebook ad un tempo

${ }^{57}$ Per la giurisprudenza della Corte europea dei diritti dell'uomo su questo problema si v. R. Petruso, Responsabilità delle piattaforme online, oscuramento di siti web e libertà di espressione nella giurisprudenza della Corte europea dei diritti dell'Uomo, in Il diritto dell'Informazione e dell'Informatica, 2018, n. 3, p. 511 ss. 
condizione e limite nel rapporto con gli utenti che chiedano l'accesso al proprio servizio.

Conseguentemente ai principi sopra esposti, l'esclusione dei ricorrenti da Facebook si pone in contrasto con il diritto al pluralismo di cui si è detto, eliminando o fortemente comprimendo la possibilità per l'Associazione ricorrente, attiva nel panorama politico italiano dal 2009, di esprimere i propri messaggi politici».

Questo provvedimento cautelare consente due tipi di rilievi. Da un lato essa invoca alcuni principi costituzionali essenziali che hanno una dimensione giusfondamentalistica, in particolare il diritto di associazione in partiti politici e ritiene che essi siano stati violati dalla misura restrittiva decisa da Facebook. Dall'altro la sentenza nega rilevanza alla natura giuridica di Facebook, vale a dire quella di soggetto privato e tratta questo operatore come se fosse un soggetto pubblico, ritenendo che esso sia tenuto a conformarsi ai suddetti «principi costituzionali essenziali», il che, nel caso di specie, vuol dire ritenere che nei suoi confronti operino direttamente i diritti fondamentali, come se si trattasse di un soggetto giuridico pubblico. Ciò che resta indefinito è la natura di Facebook come destinatario dei diritti fondamentali, vale a dire esattamente la questione di maggiore spessore teorico.

Più in generale si intravede qui il problema di «costituzionalizzare i GAFA» (Google, Apple, Facebook, Amazon, cui forse si dovrebbero aggiungere AirBnb e Uber ${ }^{58}$, ma l'elenco è puramente indicativo e destinato ad allungarsi) vale a dire i grandi soggetti privati ${ }^{59}$ che operano sulla rete. A prima vista, infatti, alcuni dei soggetti ora menzionati sono meri contenitori, di cui occorre soltanto assicurare la neutralità. Tuttavia, a parte il fatto che tale neutralità può essere attualmente messa in discussione, con la conseguenza che essa va interpretata più come un sollen che come un sein, tali soggetti sono poteri privati, capaci di incidere sulla libertà dei fruitori dei loro servizi. E la loro natura di soggetti al tempo stesso privati e transnazionali rende talora problematico assoggettarli al dovere di rispettare $\mathrm{i}$ diritti fondamentali e più in generale di non operare come se fossero legibus soluti. Al tempo stesso non mancano casi nei quali gli Stati esigono dai GAFA l'esercizio

\footnotetext{
${ }^{58}$ Su cui v. M. Midiri, Nuove tecnologie e regolazione: il «caso Uber», in Rivista Trimestrale di Diritto Pubblico, 2018, n. 3, p. 1017 ss.

${ }^{59}$ Ha osservato correttamente S. Rodotà, Una Costituzione per Internet, in Politica del diritto, 2010, n. 3 , p. 341: «Google non è soltanto una delle strapotenti società multinazionali. E' un potere a sé, superiore a quello di un'infinità di Stati nazionali, con i quali negozia appunto da potenza a potenza. E' interlocutore quotidiano di centinaia di milioni di persone, alle quali offre la possibilità di entrare e muoversi nell'universo globale. Governa corpi, conoscenze, relazioni sociali». Sia pur con i necessari adattamenti, ragionamenti simili possono essere svolti anche riguardo ad altri soggetti del web.
} 
di un potere di vigilanza ed ascrivono ad essi una responsabilità per i contenuti in essi pubblicati. Del resto, anche ammesso che Facebook e gli altri «contenitori» operanti su Internet. siano a tutti gli effetti destinatari dei diritti fondamentali, così come lo sono i poteri pubblici e che essi siano abilitati anche a limitare tali diritti allo stesso modo in cui lo sono i poteri pubblici, si imporrebbe l'esigenza di un giusto processo nelle restrizioni ai diritti che essi sono tenuti a rispettare e dell'applicazione del principio di proporzionalità nella restrizione. Ad es., nella vicenda, sopra evocata, di Casapound e degli esponenti di estrema destra italiani, ci si può chiedere se Facebook non avesse dovuto intervenire sui singoli contenuti dei post di tali soggetti piuttosto che oscurare radicalmente una serie di profili, oltretutto con criteri arbitrari (che in almeno un caso hanno condotto ad oscurare anche il profilo di un soggetto che si era espresso criticamente nei confronti dei gruppi di estrema destra).

\section{Il diritto all'oblio}

La questione del c.d. diritto all'oblio era emersa già con riferimento all'esercizio del diritto di cronaca nei tradizionali mezzi di diffusione del pensiero, come la stampa e la radiotelevisione. Il concetto di diritto all'oblio era stato elaborato inizialmente dalla dottrina civilistica, che lo aveva collocato nell'ambito dei diritti della personalità. Esso era stato inizialmente inteso come «diritto di un soggetto a non vedere pubblicate notizie relative a vicende, già legittimamente pubblicate, rispetto all' accadimento delle quali è trascorso un notevole lasso di tempo» ${ }^{60} \mathrm{La}$ Corte di Cassazione aveva riconosciuto tale diritto ravvisando l'illiceità della ripubblicazione di notizie astrattamente lesive della reputazione e dell'onore della persona qualora facciano difetto nuovi elementi che giustifichino nel presente la ripubblicazione di notizie riferite al passato ${ }^{61}$. In questi casi, quindi, il diritto all'oblio è un riflesso dell'assenza di un interesse attuale alla notizia che escluderebbe una invasione della riservatezza.

«Il diritto all'oblio è mutato con l'utilizzo di Internet e delle reti telematiche, per le modalità proprie di diffusione dell'informazione. Infatti in Rete la ripubblicazione non è necessaria, dal momento che per la stessa organizzazione dell'informazione nella Rete, l'informazione non è cancellabile, ma permane disponibile» ${ }^{62}$. Il diritto all'oblio, pertanto, assume una configurazione in parte differente, e non si traduce in un divieto di ripubblicazione di notizie già pubblicate, ma nell'obbligo di correggere notizie o di ridurne la reperibilità; inoltre il principale destinatario di questo diritto non è più o non è soltanto il giornalista, ma il motore di ricerca, che, trattando in forma automatizzata i dati personali di un certo soggetto interessato, consente al pubblico di prendere conoscenza di una

\footnotetext{
${ }^{60}$ G. Finocchiaro, Il diritto all'oblio, in Il diritto dell'informazione dell'informatica, 2014, p. 592.

${ }^{61}$ Cass. Civ., sez. III, sent. n. 3679/1998.

${ }^{62}$ G. Finocchiaro, Il diritto all'oblio, cit., p. 593.
} 
notizia pregiudizievole anche a distanza di tempo, quando ormai sia del tutto cessata l'attualità della notizia.

Nella dottrina italiana, chi si è occupato del tema con uno studio monografico ha inteso il diritto all'oblio come diritto ad una privacy storica, vale a dire a una rimozione delle notizie pregiudizievoli per la vita privata e per la reputazione della persona che abbiano perduto attualità ${ }^{63}$. Si è molto discusso della sua estensione e della sua esatta configurazione ${ }^{64} \mathrm{e}$, in assenza di un espresso riconoscimento legislativo, si è inizialmente fatto leva su alcune disposizioni del Codice della privacy, che - in attuazione della Direttiva europea 95/46/CE - consentono la cancellazione, la rettificazione, l'integrazione o l'anonimizzazione di dati personali

${ }^{63}$ M. Mezzanotte, Il diritto all'oblio. Contributo allo studio della privacy storica, Edizioni Scientifiche Italiane, Napoli, 2009.

${ }^{64} \mathrm{E}$. Gabrielli (a cura di), Il diritto all'oblio, Napoli, 1999; T. Auletta, Diritto alla riservatezza e "droit à l'oubli", in G. Alpa, M. Bessone, L. Boneschi, G.D. Caiazza, L'informazione e $i$ diritti della persona, Napoli, 1983, p. 127 ss.; G.B. Ferri, Diritto all'informazione e diritto all'oblio, in Riv. Dir. Civ., 1990, p. 801 ss.; M.R. Morelli, Oblio (diritto all'), in Enciclopedia del diritto, Aggiornamento, vol. VI, Giuffré Milano, 2002; S. Niger, Diritto all'oblio, in G. Finocchiaro (a cura di), Diritto all'anonimato. Anonimato, Nome e identità personale, Cedam, Padova, 2007; D. Messina, Le prospettive del diritto all'oblio nella società dell'informazione e della comunicazione, in Informatica e diritto, 2009, p. 93-103; R. Pardolesi, F. Di Ciommo, Dal diritto all'oblio in Internet alla tutela dell'identità dinamica. E' la rete, bellezza!, in Danno e responsabilità, 2012; L. Ferola, Dal diritto all'oblio al diritto alla memoria sul web. L'esperienza applicata italiana, in Dir. Inf., 2012; L. De Grazia, La libertà di stampa e il diritto all'oblio nei casi di diffusione di articoli attraverso internet: argomenti comparativi, in Rivista AIC, 2013, n. 4; G. Finocchiaro, Il diritto all'oblio nel quadro dei diritti della personalità, in Il diritto dell'informazione dell'informatica, 2014, p. 591-604; A. Salerno, Il diritto all'oblio nella più recente giurisprudenza, in Giustizia Civile, 2014; G.M. Riccio, Diritto all'oblio e responsabilità dei motori di ricerca, in Il diritto dell'informazione e dell'informatica, 2014, p. 753-773; A. Palmieri, R. Pardolesi, Dal diritto all'oblio all'occultamento in rete: traversie dell'informazione ai tempi di Google, in Nuovi quaderni del Foro italiano, 1, 2014.; A. Salarelli, Diritto all'oblio e archivi online dei quotidiani: alcune considerazioni sulla memoria sociale ai nostri tempi, in Italian Journal of Library, Archives, and Information Science, jan. 2014; A.L. Valvo, Il diritto all'oblio nell'epoca dell'informazione "digitale", in Studi sull'integrazione europea, 2015, p. 347-357; G. Resta, V. Zeno Zencovich, Il diritto all'oblio su Internet dopo la sentenza Google Spain, Roma Tre Press, Roma, 2015; F. Sassano, Il diritto all'oblio fra Internet e mass media, Editore Key, Vicalvi, 2015; T.E. Frosini, Google e il diritto all'oblio preso sul serio, in Diritto dell'informazione e dell'informatica, 2014, n. 4-5; M. Cocuccio, Il diritto all'oblio fra tutela della riservatezza e diritto all'informazione, in Diritto di famiglia e delle persone, 2015, 2; G. Miniussi, Il "diritto all'oblio": i paradossi del caso "Google", in Rivista italiana di diritto pubblico comunitario, 2015 n. 1; G. Citarella, "Diritto all'oblio" e rilevanza del tempo, in Responsabilità Civile e Previdenza, 2016, n. 2; A. Mantelero, "Right to be forgotten" e pubblici registri, in Nuova giurisprudenza civile commentata, 2016, n.1; M. Zanicheli, Il diritto all'oblio tra privacy e identità digitale, in Informatica e diritto, 2016, n. 1, p. 9-28; S. Martinelli, Diritto all'oblio e motori di ricerca: il bilanciamento tra memoria e oblio in internet e le problematiche poste dalla de-indicizzazione, in Il diritto dell'Informazione e dell'Informatica, 2017, n.3, p. 565 ss.; M. Tampieri, Il diritto all'oblio e la tutela dei dati personali, in Responsabilità civile e previdenza, 2017, n. 3, p. 1010 ss.; F. Barra Caracciolo, La tutela della personalità in Internet, in Il diritto dell'Informazione e dell'Informatica, 2018, n. 2, p. 201 ss. 
trattati in violazione di legge ${ }^{65}$. Ovviamente la famosa sentenza Google Spain della Corte di Giustizia dell'Unione europea ha rappresentato un punto di svolta nel dibattito. Ma la dottrina italiana ${ }^{66}$ ha notato che tale sentenza ha riconosciuto solo in parte il diritto all'oblio, vale a dire il c.d. diritto alla deindicizzazione o de-listing di informazioni che risultino nocive per la dignità della persona e non siano più di interesse pubblico. In una decisione successiva, la Corte di giustizia ha poi precisato che l'obbligo di rimozione per il motore di ricerca vale solo sul territorio dell'Unione europea.

La stessa dottrina ha sottolineato i problemi che restano aperti, riguardo alla tutela delle persone pubbliche, alla ricerca di un punto di equilibrio fra interesse pubblico alla notizia e dignità della persona e più in generale alla tutela della web reputation (diritto di replica o rettifica, ricerca di strumenti) ${ }^{67}$.

\section{Algoritmi, big data e provvedimenti amministrativi automatizzati}

«La giurisprudenza amministrativa si è trovata negli ultimi anni a decidere di una serie di questioni che hanno un elemento in comune: provvedimenti della pubblica amministrazione determinati da sistemi di decisione automatica guidati da algoritmi ${ }^{6}{ }^{6}$. L'uso di algoritmi da parte della pubblica amministrazione per adottare decisioni amministrative (parzialmente o interamente) automatizzate è entrato prepotentemente nelle cronache italiane con la riforma della scuola voluta

${ }^{65}$ Art. 7 del decreto legislativo n. 196/2003. M. Betzu, Regolare Internet, cit., p. 167-169. Si v. ora l'art. 17 del Regolamento Generale sulla Protezione dei Dati Personali.

${ }^{66} \mathrm{Si} \mathrm{v}$. in particolare F. Pizzetti (a cura di), Internet e la tutela della persona. Il caso del motore di ricerca, Passigli Editori, Firenze, 2015.

${ }^{67} \mathrm{Si}$ v. ancora F. Pizzetti (a cura di), Internet e la tutela della persona, cit.

${ }^{68}$ A. Simoncini, Profili costituzionali della amministrazione algoritmica, in Rivista trimestrale di diritto pubblico, 2019, n. 4, p. 1152. Si v. anche AGID - Agenzia per l'Italia Digitale, Libro bianco sull'intelligenza artificiale al servizio del cittadino, Roma, versone 1.0 marzo 2018, in https://ia.italia.it/assets/librobianco.pdf. Su questo tema si v. inoltre A. Moretti, Algoritmi e diritti fondamentali della persona. Il contributo del Regolamento (UE) 2016/679, in Il diritto dell'Informazione e dell'Informatica, 2018, n. 4, p. 799 ss.; G. Resta, Governare l'innovazione tecnologica: decisioni algoritmiche, diritti digitali e principio di uguaglianza, in Politica del diritto, 2019, n. 2, p. 199-236; L. Viola, L'intelligenza artificiale nel procedimento e nel processo amministrativo: lo stato dell'arte, in Il Foro Amministrativo, 2018, n. 9, p. 1598 ss.; S. Crisci, Intelligenza artificiale ed etica dell'algoritmo, in Il Foro Amministrativo, 2018, n. 10, p. 1787 ss.; R. Ferrara, Il giudice amministrativo e gli algoritmi. note estemporanee a margine di un recente dibattito giurisprudenziale, in Diritto Amministrativo, 2019, n. 4, p. 773 ss.; S. Scalzini, Alcune questioni a proposito di algoritmi, dati, etica e ricerca, in Rivista Italiana di Medicina Legale (e del Diritto in campo sanitario), 2019, n.1, p. 169 ss.; C. Casonato, Intelligenza artificiale e diritto costituzionale: prime considerazioni, in Diritto Pubblico comparato ed europeo, 2019, p. 101 ss.; C. Faralli, Diritto, diritti e nuove tecnologie, Editoriale Scientifica, Napoli, 2018; A. Carleo (a cura di), La decisione robotica, Il Mulino, Bologna, 2019; G. Avanzini, Decisioni amministrative e algoritmi informatici. Predeterminazione, analisi predittiva e nuove forme di intelligibilità, Editoriale Scientifica, Napoli, 2019. 
dal governo Renzi (c.d. «Buona scuola») ${ }^{69}$, nell'implementazione della quale il governo ha proceduto al bando di un grande concorso per il reclutamento in forma stabile di insegnanti nella scuola secondaria, al fine di stabilizzare una parte importante del personale scolastico, a lungo collocato in una situazione di precariato. In questo contesto, il caso più controverso di utilizzazione di algoritmi è stato quello della assegnazione degli insegnanti vincitori del concorso alla loro prima sede di servizio.

La questione dell'assegnazione degli insegnati alle rispettive sedi di servizio era resa complicata dallo storico squilibrio esistente in Italia fra le Regioni dalle quali proviene un elevato numero di insegnanti (per lo più le Regioni dell'Italia meridionale) - Regioni nelle quali in alcuni casi tali insegnanti prestavano servizio in forma non stabilizzata prima di vincere il concorso - e quelle in cui esiste un maggior numero di posti disponibili (le Regioni dell'Italia centrale e settentrionale). Tale squilibrio genera da un lato il desiderio degli insegnanti di essere assegnati alla Regione di origine, o a una sede non eccessivamente distante da essa, e dall'altro l'insoddisfazione delle famiglie e degli studenti delle Regioni dell'Italia settentrionale per la permanenza solo temporanea nella loro sede dei docenti assegnati a tali Regioni.

Un algoritmo è stato utilizzato per assegnare la sede agli insegnanti vincitori di concorso, con la finalità di rispettare il principio di imparzialità dell'amministrazione pubblica e di utilizzare criteri il più possibile oggettivi - a garanzia dell'eguaglianza dei cittadini davanti alla legge - e al tempo stesso per velocizzare il procedimento decisionale. I risultati, tuttavia, hanno generato diffusa insoddisfazione nei docenti assegnati a sedi molto lontane da quelle di origine. Alcuni di tali docenti ed i loro sindacati hanno di conseguenza richiesto ${ }^{70} \mathrm{l}^{\prime}$ accesso agli atti in base ai quali l'assegnazione della sede era stata effettuata, ma il Ministero dell'Università ha ritenuto di non poter rendere noto l'algoritmo sulla base del quale la decisione era stata presa, in ragione del diritto di proprietà intellettuale degli autori dell'algoritmo (predisposto da una società privata, esterna al Ministero).

Il Tribunale Amministrativo regionale (TAR) del Lazio, davanti al quale i docenti hanno impugnato l'atto del Ministero dell'Università, nell'annullare gli atti di diniego all'accesso all'algoritmo, ha invocato alcuni importanti principi che, pur avendo origine nel diritto amministrativo, hanno uno spessore costituzionale: il destinatario di una decisione amministrativa automatica, adottata in base ad un algoritmo, ha diritto a conoscere il «codice sorgente», vale a dire il criterio seguito dall'algoritmo per produrre la decisione ${ }^{71}$. Inoltre, mentre in alcune decisioni il ricorso ad un algoritmo è stato ritenuto ammissibile solo in procedimenti

\footnotetext{
${ }^{69}$ Si tratta della legge n. 107/2015.

${ }^{70}$ In base all'art. 22 della 1. n. 241/1990.

${ }^{71}$ TAR Lazio, Sezione III-bis, 14.2.2017, n. 3769, pubblicata in Giornale di diritto amministrativo, 2018, p. 647, con nota di I Forgione, Il caso dell'accesso al sofware MIUR per l'assegnazione dei docenti, ivi).
} 
amministrativi nei quali l'amministrazione è vincolata alla decisione, ma non in quelli nei quali essa gode di discrezionalità, in altre si è ritenuto che l'utilizzazione di un algoritmo per adottare una decisione amministrativa che può incidere restrittivamente nella sfera giuridica del soggetto privato non può mai sostituire l'attività valutativa che deve essere svolta da un funzionario amministrativo persona fisica:

«le procedure informatiche, finanche ove pervengano al loro maggior grado di precisione, e addirittura di perfezione, non possono mai soppiantare, sostituendola davvero appieno, l'attività cognitiva, acquisitiva e di giudizio che solo un'istruttoria affidata ad un funzionario persona fisica è in grado di svolgere e che pertanto ... deve seguitare ad essere il dominus del procedimento stesso, all'uopo dominando le stesse procedure informatiche predisposte in funzione servente e alle quali va tutt'oggi riservato un ruolo strumentale e meramente ausiliario in seno al procedimento amministrativo e giammai dominante o del tutto surrogatorio dell'attività dell'uomo ... $\gg^{72}$.

Il TAR Lazio ha appoggiato questa sua affermazione - condivisibile a nostro avviso su un piano etico-politico - sui «valori costituzionali scolpiti negli art. 3, 24 e 97 della Costituzione, oltre che dell'art. 6 della Convenzione europea dei diritti dell'uomo». Dunque ha invocato, rispettivamente il principio di eguaglianzaragionevolezza-proporzionalità, il diritto alla difesa, i principi di buon andamento e di imparzialità dell'amministrazione pubblica e il diritto ad un giusto processo. Ora, se si può accettare che alcuni profili di tali principi costituzionali siano in tensione con 1'uso degli algoritmi nella pubblica amministrazione, quest'ultimo può a sua volta fondarsi proprio su alcuni di quegli stessi principi, essendo sicuramente servente rispetto al buon andamento (efficienza) e all'imparzialità dell'amministrazione (art. 97 Cost. italiana) e allo stesso principio costituzionale di eguaglianza (art. 3 Cost. italiana). La base costituzionale invocata dal TAR Lazio appare dunque piuttosto fragile e in effetti l'assolutezza di alcune affermazioni contenute in alcune delle sue decisioni è stata in seguito parzialmente ridimensionata dal Consiglio di Stato ${ }^{73}$, il quale, comunque, pur non escludendo la decisione amministrativa algoritmica, ritiene che essa debba essere consentita solo nei casi di amministrazione vincolata. Essa andrebbe invece esclusa qualora l'attività amministrativa sia discrezionale: ove la legge lascia all'amministrazione un potere decisionale, quest'ultimo deve essere esercitato da un funzionario amministrativo umano e non da un meccanismo automatizzato.

72 TAR Lazio, Sezione III-bis n. 9224-9230/2018.

${ }^{73}$ Si v. la sentenza Cons. St., VI sez., 8.4.2019, n. 2770. Sulle differenti posizioni del TAR Lazio e del Consiglio di Stato si v. A. Simoncini, Profili costituzionali, cit., p. 1163. 
Resta il problema di trovare un fondamento adeguato per questo emergente diritto a non essere pregiudicato da una decisione amministrativa esclusivamente automatica. Il richiamo del TAR Lazio ai «valori costituzionali scolpiti negli art. 3, 24 e 97 della Costituzione, oltre che dell'art. 6 della Convenzione europea dei diritti dell'uomo» appare, come si è detto, troppo generico, oltre ad evocare il discutibile metodo dell'interpretazione per valori.

Un fondamento alternativo può essere ricercato nel diritto europeo in materia di protezione dei dati personali ${ }^{74}$, da cui sono desumibili almeno tre principi applicabili a questo tipo di casi: il principio di non esclusività, vale a dire il diritto ad un intervento umano nel processo decisionale, il principio di conoscibilità (che dà diritto al destinatario di conoscere le tecniche utilizzate per la decisione) e di comprensibilità (il destinatario della decisione ha diritto a una spiegazione in linguaggio comprensibile dei criteri utilizzati) e il principio di non discriminazione. In particolare, l'art. 22 del GDPR, infatti, stabilisce che «l'interessato ha il diritto di non essere sottoposto a una decisione basata esclusivamente sul trattamento automatizzato, compresa la profilazione, che produca effetti giuridici che lo riguardano o che incida in modo analogo significativamente sulla sua persona». Tuttavia, pur proclamando tale diritto, il GDPR consente una serie di eccezioni, che possono fondarsi su tre diverse esigenze: a) la necessità di concludere o eseguire un contratto; b) la previsione dell'eccezione da parte del diritto dell'Unione o dello Stato membro; c) il consenso dell'interessato. Ne risulta dunque una serie corposa di eccezioni, che invece, secondo il giudice amministrativo italiano, non sarebbero ammissibili, anzitutto sulla base dei principi che reggono il procedimento amministrativo.

Ne risulterebbe, comunque, un nuovo diritto a non essere oggetto di decisioni automatiche, di cui resta da stabilire se possa essere inteso come un vero e proprio diritto fondamentale e di cui la dottrina e la giurisprudenza dovranno indagare nei prossimi anni l'esatto fondamento costituzionale, oltre a costruire un framework concettuale che consenta di individuarne i limiti e di costruire adeguate forme di bilanciamento. Al momento, si può ipotizzare che il principio secondo cui «la tecnologia ... deve rimanere nell'ambito dei mezzi e degli strumenti e non può mai sostituirsi alla decisione umana, quantomeno ogniqualvolta la decisione sia suscettibile di incidere le libertà e i diritti», vada ricercato nell' essenza della nozione di dignità umana, in senso kantiano ${ }^{75}$, vale a dire nell'obbligo di considerare l'essere umano sempre come un fine e mai come un mezzo.

A maggior ragione, queste considerazioni vanno tenute presenti quando si sposta il discorso dalla decisione amministrativa a quella giurisdizionale. Su questo

\footnotetext{
${ }^{74} \mathrm{Si}$ v. la ricostruzione di A. Simoncini, L'algoritmo incostituzionale, in Rivista di bio-diritto/Bio Law Journal, 2019, n. 1, p. 77 ss.

75 A. Simoncini, Profili costituzionali, cit., p. 1186.
} 
tema la riflessione della dottrina italiana è ancora agli inizi, ma alcune linee sono state già tracciate ${ }^{76}$ e dovranno essere ulteriormente sviluppate nei prossimi anni.

\section{Una "dichiarazione dei diritti in Internet"?}

Durante la XVII legislatura repubblicana (2013-2018), la presidente della Camera dei Deputati Laura Boldrini ha nominato una commissione di studio che ha elaborato una bozza di «Dichiarazione dei diritti su Internet», che è stata sottoposta a consultazione pubblica e poi pubblicata, sul sito web della Camera. Il testo prodotto da tale commissione ${ }^{77}$, che aspira ad essere qualcosa di simile a ciò che in Brasile è il Marco Civil da Internet. non è stato tradotto in un atto normativo, ma verrà qui brevemente analizzato in quanto esso tenta di offrire risposte a molti dei problemi cui si è accennato nei paragrafi precedenti. Ovviamente queste pretese regolative possono sembrare inadeguate, per due fondamentali ragioni: da un lato la natura civilistica di internet (c.d. Internet governance by contract) e dall'altro l'incompatibilità di una dichiarazione dei diritti nazionale con la struttura transnazionale del mezzo Internet ${ }^{78}$.

L'art. 1 di questa dichiarazione fa rinvio ai diritti fondamentali già riconosciuti dalla Dichiarazione universale dei diritti umani, dalla Carta dei diritti dell'Unione europea, dalle dichiarazioni internazionali in materia e dalle Costituzioni nazionali per ribadirne la vigenza rispetto ad Internet. Si tratta di un rinvio molto generico, che mette sullo stesso piano documenti dal valore giuridico assai diverso e che, oltretutto, richiama in genere le costituzioni nazionali, senza distinguere quelle straniere da quella italiana, e, fra le prime, neppure le Costituzioni autoritarie da quelle liberal-democratiche. L'art. 1 prevede poi due criteri interpretativi relativamente a tali diritti: da un lato essi devono essere interpretati in modo da favorirne l'effettività; dall'altro i bilanciamenti fra i diversi diritti devono essere imperniati sul pieno rispetto della dignità, della libertà, dell'eguaglianza e della diversità di ogni persona, «che costituiscono i principi in base ai quali si effettua il

\footnotetext{
${ }^{76}$ Si v. M. Luciani, Una decisione giudiziaria robotica, in Rivista AIC, 3/2018, pubblicato il 30.09.2018.

$77 \mathrm{Si} \mathrm{v}$. il testo di tale dichiarazione, accompagnato da alcune note illustrative, in https://www.camera.it/application/xmanager/projects/leg17/commissione internet/dichiarazione dei diritti internet pubblicata.pdf. Si v. il commento di T. Natoli, La 'Dichiarazione dei diritti in Internet' italiana: una prospettiva internazionalista, in http://www.sidiblog.org/2015/09/24/ladichiarazione-dei-diritti-in-internet-italiana-una-prospettiva-internazionalista/

${ }^{78}$ Rileva A. Oddenino, Code is still law: la codificazione dei diritti in Internet, la tutela dei dati personali e l'arduo contrappunto del diritto alla tecnocrazia, in http://www.sidiblog.org/2015/10/12/code-is-stilllaw-la-codificazione-dei-diritti-in-internet-la-tutela-dei-dati-personali-e-larduo-contrappuntodel-diritto-alla-tecnocrazia/, che si tratta di un assetto internazionale pre-wetfaliano, fortemente asimmetrico, con dominio di enti connessi al governo degli Stati Uniti.
} 
bilanciamento con altri diritti»: dove non è affatto chiaro che cosa si voglia dire circa le modalità del bilanciamento.

Mentre l'art. 1 tenta di esportare nella Rete i diritti già riconosciuti in via generale, gli articoli da 2 a 14 prevedono invece una serie di nuovi diritti relativi ad Internet.

L'art. 2 riconosce il diritto di accesso ad Internet e impegna le istituzioni al superamento del Digital divide. In particolare, esso precisa che «ogni persona ha eguale diritto di accedere a Internet in condizioni di parità, con modalità tecnologicamente adeguate e aggiornate che rimuovano ogni ostacolo di ordine economico e sociale».

L'art. 3 prevede il diritto alla conoscenza e all'educazione in rete, con l'obiettivo di favorire l'uso consapevole di Internet. $\mathrm{Il}^{\circ}$ comma, in particolare, sottolinea che «ogni persona ha diritto ad essere posta in condizione di acquisire e di aggiornare le capacità necessarie ad utilizzare Internet in modo consapevole per l'esercizio dei propri diritti e delle proprie libertà fondamentali».

L'art. 4 stabilisce il principio della net neutrality: «Ogni persona ha il diritto che i dati trasmessi e ricevuti in Internet non subiscano discriminazioni, restrizioni o interferenze in relazione al mittente, ricevente, tipo o contenuto dei dati, dispositivo utilizzato, applicazioni o, in generale, legittime scelte delle persone». Quest'ultima formula appare eccessivamente amplia e generica.

Gli art. da 5 a 10 sono dedicati alla tutela dell'individualità della persona in rete. In particolare è ribadito il diritto alla protezione dei dati personali «per garantire il rispetto della sua dignità, identità e riservatezza», da cui discendono «i principi di necessità, finalità, pertinenza, proporzionalità» nel trattamento dei dati e la necessità del consenso dell'interessato, che, però, «non può costituire una base legale per il trattamento quando vi sia un significativo squilibrio di potere tra la persona interessata e il soggetto che effettua il trattamento» (art. 5). L'art. 6 proclama il diritto all'autodeterminazione informativa, ovvero il diritto all'accesso e alla rettifica dei dati riguardanti la persona. L'art. 7 dichiara inviolabili i sistemi e i dispositivi informatici di ogni persona e la libertà e segretezza delle sue informazioni e comunicazioni elettroniche. L'art. 8 esclude che un atto o provvedimento amministrativo o giudiziario o una decisione che possa incidere sensibilmente nella sfera giuridica della persona possa essere adottato esclusivamente sulla base di un trattamento automatizzato dei dati personali. Sono inoltre previsti i diritti all'identità digitale, vale a dire «alla rappresentazione integrale e aggiornata delle proprie identità in Rete», che «riguarda la libera costruzione della personalità e non può essere sottratta all'intervento e alla conoscenza dell'interessato» (art. 9) e alla protezione dell'anonimato, con la precisazione che «limitazioni possono essere previste solo quando siano giustificate dall'esigenza di tutelare rilevanti interessi pubblici e risultino necessarie, proporzionate, fondate sulla legge e nel rispetto dei caratteri propri di una società democratica» (art. 10). 
L'art. 11 riconosce il diritto all'oblio, precisando che «ogni persona ha diritto di ottenere la cancellazione dagli indici dei motori di ricerca dei riferimenti ad informazioni che, per il loro contenuto o per il tempo trascorso dal momento della loro raccolta, non abbiano più rilevanza pubblica», ma anche che tale diritto non può limitare la libertà di ricerca, né il diritto all'informazione.

L'art. 12 prevede l'obbligo dei responsabili delle piattaforme digitali di comportarsi con lealtà e correttezza nei confronti di consumatori ed utenti e detta alcuni principi sulle informazioni e sulle condizioni generali di contratto e sulle relative modificazioni.

L'art. 13 è relativo alla sicurezza in rete delle singole persone e prevede la garanzia dell'integrità delle infrastrutture e la loro tutela da attacchi. Afferma enfaticamente che la libertà di manifestazione del pensiero in rete non incontra limiti, ma subito dopo li richiama in forma non poco intensa precisando che «deve essere garantita la tutela della dignità delle persone da abusi connessi a comportamenti quali l'incitamento all'odio, alla discriminazione e alla violenza». Verrebbero così giustificate limitazioni alla libertà di pensiero basate unicamente sul loro contenuto, nella prospettiva dei c.d. «discorsi di odio».

L'art. 14 muove da una enfatica dichiarazione secondo cui «ogni persona ha diritto di vedere riconosciuti i propri diritti in Rete sia a livello nazionale che internazionale» e richiama le regole universali e internazionali relative ad Internet.

Si tratta di una dichiarazione molto interessante dal punto di vista culturale, in quanto tentativo di codificare $i$ «nuovi» diritti necessari nell'era di Internet. La sua tecnica redazionale si presta però a varie obiezioni, dato che essa rimane un po' a metà strada fra un semplice elenco di diritti e una disciplina più tecnica: si capisce il bisogno di concretizzazione, ma alcuni aspetti più tecnici rischiano di diventare rapidamente obsoleti, senza arrivare a quel grado di dettaglio che li rende idonei ad essere autoapplicativi.

Nella dottrina italiana, comunque, la tesi favorevole a una Dichiarazione formale (dunque ad una scrittura) dei diritti su Internet non è accettata unanimemente: alcuni autori sono invece favorevoli a una utilizzazione dottrinale e giurisprudenziale delle clausole generali sui diritti contenute nella Costituzione, da utilizzare come base per riconoscere in via pretoria i diritti su Internet.

Più in generale, si è sviluppato in Italia un ricco dibattito su una «Costituzione per Internet», che ha avuto fra i protagonisti il civilista Stefano Rodotà ${ }^{79}$, già garante per la protezione dei dati personali, e sulla regolazione giuridica di Internet, circa la quale si sono pronunciati alcuni studiosi di diritto costituzionale ${ }^{80}$.

\section{Il Codice dell'amministrazione digitale}

\footnotetext{
${ }^{79}$ S. Rodotà,Una Costituzione per Internet, in Politica del diritto, 2010.

${ }^{80}$ M. Betzu, Regolare Internet, cit.; G. De Minico, Internet. Regola e anarchia, Jovene, Napoli, 2012.
} 
Parallelamente a questo dibattito, si è sviluppata una legislazione che presenta significativi riflessi giusfondamentalistici. In particolare, il codice dell'amministrazione digitale ${ }^{81}$ ha riconosciuto per i rapporti fra cittadino e pubblica amministrazione una serie di diritti paralleli a quelli preesistenti, da esercitarsi nel campo digitale: in alcuni casi, si tratta di diritti corrispondenti a quelli previsti nella Dichiarazione appena citata, da cui essi si differenziano da un lato in quanto sono norme vigenti di diritto positivo, dall'altro perché hanno una base esclusivamente legislativa e non possono dunque essere qualificati come diritti fondamentali.

Il Codice dell'amministrazione digitale è suddiviso in nove «capi». Il primo («Principi generali») contiene, oltre ad alcune definizioni, un catalogo di «diritti dei cittadini e delle imprese» e alcune norme sull'organizzazione delle pubbliche amministrazioni e sui rapporti fra Stato, regioni e autonomie locali; il secondo disciplina il documento informatico e le firme elettroniche; il terzo si occupa della formazione, gestione e conservazione dei documenti informatici; il quarto regola la trasmissione informatica dei documenti; il quinto è relativo ai dati delle pubbliche amministrazioni e ai servizi in rete; il sesto disciplina lo sviluppo, l'acquisizione e il riuso di sistemi informatici nelle pubbliche amministrazioni, il settimo contiene alcune regole tecniche; $1^{\prime}$ ottavo riguarda il sistema pubblico di connettività, mentre il nono è dedicato alle disposizioni transitorie e finali.

L'art. 3 del Codice riconosce un diritto generale di usare, in modo accessibili ed efficace, le tecnologie informatiche per l'esercizio dei diritti di accesso e di partecipazione al procedimento amministrativo. L'art. 3-bis riconosce il c.d. domicilio digitale. L'art. 7 prevede il diritto a servizi on-line semplici e integrati. L'art. 8 impegna le pubbliche amministrazioni a promuovere iniziative volte a favorire la diffusione della cultura digitale fra i cittadini, con particolare riguardo ai minori e alle categorie a rischio di esclusione. L'art. 8-bis impegna le pubbliche amministrazioni a favorire la disponibilità di connettività alla rete Internet presso gli uffici pubblici e gli altri luoghi pubblici, in particolare nei settori scolastico, sanitario e di interesse turistico. L'art. 9 stabilisce che le pubbliche amministrazioni «favoriscono ogni forma di uso delle nuove tecnologie per promuovere una maggiore partecipazione dei cittadini, anche residenti all'estero, al processo democratico e per facilitare l'esercizio dei diritti politici e migliorare la qualità dei propri atti, anche attraverso l'utilizzo, ove previsto e nell'ambito delle risorse disponibili a legislazione vigente, di forme di consultazione preventiva per via telematica sugli schemi di atto da adottare».

${ }^{81}$ Decreto legislativo n. 82/2005, e successive modificazioni, in particolare quelle apportate dal decreto legislativo n. 179/2016 e dal decreto legislativo n. 217/2017. Al riguardo si v. G. Cassano, C. Giurdanella (a cura di), Il Codice della pubblica amministrazione digitale. Commentario al D. Lgs. $n$. 82 del 7 marzo 2005, Giuffrè, Milano, 2005; I. Macrì, U. Macrì, G. Pontevolpe (a cura di), Il nuovo Codice della amministrazione digitale, Giuffrè, Milano, 2011. 


\section{Internet e la partecipazione politica.}

Oltre che sui diritti di libertà e sui diritti sociali, la rivoluzione digitale ha influito in maniera non marginale sui diritti politici e, mediante, essi, sulle istituzioni democratiche.

A) Il dibattito sull'impatto di Internet sulla politica e sulle istituzioni democratiche e autoritarie - si è articolato almeno in due grandi fasi.

Nella prima si è posto l'accento sulle nuove opportunità aperte per la partecipazione democratica da Internet, anzitutto come strumento di «difesa della democraticità di un sistema politico» ${ }^{82}$ (disarticolando la segretezza dei documenti e delle comunicazioni governative o facilitando l'organizzazione di movimenti di protesta e di opposizione a regimi politici autoritari). Si è sottolineato che i social media hanno contribuito in maniera non marginale a indebolire regimi autoritari che parevano inossidabili, e ciò pur in assenza di organizzazioni o leadership forti: si pensi per tutti al caso delle Primavere arabe.

Accanto a questa pars destruens, è venuta emergendo anche una potenzialità costruttiva di Internet, che si è delineato come un mezzo per ridurre le barriere di accesso all' ampliamento dell'offerta politica. Esso ha contribuito alla formazione in tempi molto rapidi di forze politiche nuove, che hanno utilizzato la rete come supporto principale della loro organizzazione: si pensi ai casi dei vari "partiti pirata" in Germania e in Svezia, al Movimento Cinquestelle in Italia dal 2013 in poi, alla stessa creazione di La Republique en Marche e, prima di essa, della candidatura Macron fra il 2016 ed il 2017 in Francia.

Ben presto, tuttavia, la rete è stata utilizzata anche con forme, contenuti e finalità da molti ritenuti pericolosi per le istituzioni rappresentative. $\mathrm{E}^{\prime}$ così subentrata una seconda fase, molto più problematica di quanto gli entusiasmi iniziali potevano far supporre: il 2016 è stato forse l'anno della presa di coscienza della natura problematica dell'uso di Internet e in particolare dei social media, in occasione di una serie di consultazioni referendarie ed elettorali, come il referendum sulla permanenza o sull'uscita della Gran Bretagna dall'Unione europea; il referendum sull'accordo di pace fra il governo e le FARC in Colombia; il referendum sulla riforma costituzionale in Italia e l'elezione di Donald Trump alla presidenza degli Stati Uniti. L'esplosione dei populismi, ben visibile in occasione di tali consultazioni, ha indotto molti osservatori ad evidenziare (con un certo ritardo, forse) i rischi per la democrazia posti dall'uso dei social media, attorno alle parole chiave delle fake news ${ }^{83}$ e della post-truth. In altre parole, il secondo decennio del XX secolo ha visto l'emersione drammatica del lato problematico di Internet e in particolare dei social media e ha spento il clima di ottimismo dominante all'inizio

\footnotetext{
82 G. Azzariti, Internet e Costituzione, in Politica del diritto, 2011, n. 3, p. 369.

83 P. Bernal, Facebook: Why Facebook Makes the Fake News Problem Inevitable - Caught in the Web: Capturing the Zeitgeist of Big Tech Companies, Social Media Speech and Privacy, in Northern Ireland Legal Quarterly, Vol. 69, Issue 4 (Winter 2018), pp. 513-530.
} 
del decennio stesso, nel contesto, ad es., delle "primavere arabe» e del caso Wikileaks.

In Italia il punto di emersione dell'antipolitica telematica è stato il referendum del 4 dicembre 2016 sulla riforma costituzionale approvata pochi mesi prima dal Parlamento italiano e fortemente sostenuto fortemente dal governo italiano dell'epoca e che l'elettorato ha respinto nettamente in un clima dominato dall'antipolitica, comparabile a quelli manifestatisi nelle altre consultazioni popolari svoltesi nel 2016 e prima citate.

B) La valenza aperta, partecipativa e interattiva ${ }^{84} \mathrm{di}$ Internet era stata sottolineata sin dagli anni novanta dalla Corte suprema degli Stati Uniti, la quale l'ha qualificato come «la forma di comunicazione di massa più partecipativa che sia stata finora realizzata» ${ }^{85}$.

Tuttavia nell'analisi dell'impatto di Internet sulle istituzioni democratiche (e sull'esercizio del diritto fondamentale di voto, che le innerva) si possono identificare almeno due grandi scuole di pensiero, che chiameremo, per comodità, minimalista e massimalista:

a) quella che ha visto in Internet nuove forme e nuovi canali (e nuovi problemi) per la legittimazione ed il funzionamento delle istituzioni democratiche, oltre a un mezzo che avrebbe potuto sfidare i regimi autoritari ${ }^{86}$;

b) quella che ha visto in Internet «uno strumento persino capace di incidere sulla forma di Stato e di governo» ${ }^{87}$, non solo rivitalizzando la democrazia rappresentativa, ma ponendo le condizioni per una democrazia diretta sino ad allora tecnicamente impossibile.

Entrambe queste scuole di pensiero colgono la profondità dell'impatto prodotto sulle procedure democratiche dalla rete, nelle varie fasi della sua evoluzione cui abbiamo sinora assistito, ma mentre la prima constata l'esistenza di novità operanti dentro il quadro organizzativo pre-esistente, la seconda prefigura un cambio di scenario nell'organizzazione della democrazia e profetizza l'inversione della

84 "Grazie a Internet oggi tutti possono essere al tempo stesso comunicatori e diffusori" (TE Frosini, Il diritto costituzionale di accesso a Internet, p. 7)

${ }^{85}$ Corte suprema USA, American Civil Liberties Union v. Reno [E.D. Pa 1996], tr. it. in Il diritto dell'informazione e dell'informatica, 1996, n. 4/5, p. 604 ss. (con nota di V. Zeno Zencovich, Manifestazione del pensiero, libertà di comunicazione e la sentenza sul caso "Internet"); con sviluppi in Corte Suprema 521 US 844 (1997), tr. it. in Foro Italiano, Parte IV-2, 1998, p. 23 ss. Cf. anche G. Ziccardi, La libertà di espressione in Internet al vaglio della Corte Suprema degli Stati Uniti, in Quaderni costituzionali, 1998, n. 1, p. 123 ss.

${ }^{86} \mathrm{Si}$ v. ad es. P. Costanzo, Quale partecipazione politica attraverso le nuove tecnologie comunicative in Italia, in Il diritto dell'informazione e dell'informatica, 2011, n. 1, p. 19 ss.; nonché Id., La «democrazia digitale» (precauzioni per l'uso), in Diritto pubblico, 2019, n. 1, p. 85-86.

${ }^{87}$ M. Villone, Internet e la politica, cit., p. 568. 
gerarchia fra democrazia rappresentativa e democrazia diretta che si è realizzata nel mondo occidentale a partire dalla fine del XVIII secolo.

Pur essendo l'Italia un Paese relativamente piccolo e ormai anche periferico rispetto alle dinamiche del potere globale e pur in assenza di riforme istituzionali di qualsiasi tipo, essa si è rivelata un vero e proprio laboratorio per misurare l'impatto di Internet sul funzionamento delle istituzioni di una democrazia consolidata. Il tema è stato intensamente discusso sia sui mezzi di comunicazione di massa, sia dagli scienziati politici e sociali ${ }^{88}$, sia dai costituzionalisti ${ }^{89}$. Il clamoroso risultato delle elezioni legislative del 2013, quando il Movimento Cinquestelle, un movimento non organizzato secondo le forme classiche dei soggetti politici, che ha ottenuto il 25 per cento dei voti, diventando la prima forza politica per voti ottenuti in Italia alla Camera dei deputati. Quella stessa forza politica è addirittura diventata il principale partito di governo nelle elezioni legislative del 4 marzo 2018 (quando ha ottenuto il 32 per cento dei voti), anche se nelle recenti elezioni europee del 23 maggio 2019 i suoi consensi si sono ridotti quasi alla metà (17 per cento). La vicenda del Movimento Cinquestelle è sicuramente di grande rilievo, ma può essere letta diversamente nella prospettiva delle due scuole di pensiero di cui si parlava prima.

Il Movimento Cinquestelle è caratterizzato da almeno quattro elementi distintivi: a) l'assenza di una organizzazione articolata sul territorio sul modello dei partiti tradizionali; b) la formazione di gruppi locali più o meno spontanei; c) la leadership carismatica di un comico, noto a causa della sua partecipazione alcuni decenni orsono a trasmissioni televisive nazionali, Beppe Grillo, cui dal 2015 si è affiancato un "capo politico", incaricato di definire la linea politica del partito; d) una società di esperti di informatica (la Casaleggio \& Associati) che gestisce un sito web, (www.beppegrillo.it), che rappresenta il principale strumento organizzativo del Movimento. Gli aderenti al Movimento possono registrarsi al sito web, attraverso il quale è loro consentito di esprimersi sulle questioni su cui il gruppo dirigente del Movimento intende consultarli. I costi di questa struttura, pur non essendo inesistenti, sono nel complesso limitati.

Nella prospettiva minimalista, la storia di questo Movimento dimostra quanto Internet abbia innovato nelle modalità di funzionamento delle istituzioni rappresentative: in particolare, Internet consente oggi di sostituire quasi del tutto l'organizzazione pesante e costosa dei vecchi partiti di massa, o di quel che ne era

\footnotetext{
${ }^{88}$ Si v. ad es. D. Pitteri, Democrazia elettronica, Laterza, Roma-Bari, 2007; G. Gometz, Democrazia elettronica. Teoria e tecnica, Edizioni ETS, Pisa, 2017; G. Fioriglio, Democrazia elettronica. Presupposti e strumenti, Wolters Kluwer, Milano, 2011; P. Otranto, Internet nell'organizzazione amministrativa. Reti di libertà, Bari, 2015; B. Carfagna (a cura di), La democrazia digitale. La seconda fase, e-book, 2018 (https://www.panorama.it/wp-content/uploads/2018/03/Democrazia-digitale-la-secondafase.pdf?x50635).

${ }^{89}$ L. Bruscuglia, R. Romboli (a cura di), Diritto pubblico e diritto privato nella rete delle nuove tecnologie, Pisa, 2010.
} 
rimasto negli ultimi anni, con una struttura leggera, imperniata prevalentemente sulla rete e sulle modalità partecipative e interattive che essa consente. Un successo come quello del Movimento Cinquestelle sarebbe stato impossibile nell'era preInternet.

Occorre però essere più prudenti nell'accogliere l'interpretazione massimalista. I dirigenti del Movimento Cinquestelle presentano sé stessi e il loro partito come portatori di una forma radicalmente nuova di fare politica, in cui sarebbero $\mathrm{i}$ cittadini a prendere direttamente tutte le decisioni politiche più importanti, mediante la Rete: selezione dei candidati alle elezioni parlamentari; scelta fra i candidati a cariche pubbliche che devono essere eletti dagli organi rappresentativi (Presidente della Repubblica, membri di autorità di garanzia, ecc.), decisione sulle alleanze fra il Movimento Cinquestelle e gli altri partiti, scelte di contenuto su questioni controverse di politica pubblica (ad es. in materia di infrastrutture) o sulle regole organizzative del Movimento (ad es. sul limite di due mandati per gli eletti). Di fronte a questo potere dei cittadini, i titolari di cariche pubbliche sarebbero meri esecutori (ad es. i capigruppo parlamentari sono stati ridenominati «portavoce») della volontà autentica del popolo. Il Parlamento sarebbe definitivamente consegnato ad un ruolo di camera di registrazione della volontà popolare (fra l'altro con la pretesa a sostituire il libero mandato parlamentare con il mandato imperativo e con il rafforzamento degli spazi di decisione popolare diretta mediante referendum rispetto al ruolo delle istituzioni rappresentative). Jean Jacques Rousseau, con duecento anni di ritardo, si sarebbe preso la definitiva rivincita sul barone di Montesquieu: e ciò proprio grazie ad Internet.

Tuttavia non è difficile smascherare il carattere ideologico di questa lettura massimalista: e ciò non solo riguardo al caso concreto del Movimento Cinquestelle in Italia, ma, più in generale, circa le diverse prospettive aperte sinora da Internet al funzionamento della democrazia. Nessuna nuova era di democrazia diretta si è sinora aperta, né in Italia, né altrove. I nuovi canali partecipativi offerti da Internet sono certo più ampi e più fruibili che in passato, ma sono utilizzati in effetti da poche migliaia di persone su milioni di elettori. Le domande cui gli internauti sono chiamati a rispondere sono pre-confezionate dai leaders del Movimento Cinquestelle e dai loro consulenti informatici. Solo una piccola parte delle questioni rilevanti nella vita del partito e nelle scelte da questo compiute negli organi rappresentativi è rimessa alla decisione degli iscritti al blog www.beppegrillo.it e solo una piccola parte di questi ultimi partecipa effettivamente all'adozione delle decisioni.

In breve: la democrazia diretta - se quella via Internet può essere qualificata come tale - resta l'eccezione e le istituzioni rappresentative, con i partiti al loro interno, rimangono la regola.

Nessuna trasformazione costituzionale fondamentale si è prodotta: solo un nuovo gruppo di potere, più capace nell'uso delle tecniche di comunicazione, è riuscito a diventare governo, mandando all'opposizione i governanti precedenti. 
Mi pare che si possa dire lo stesso sulla - certo molto diversa - vicenda del Presidente Bolsonaro in Brasile.

\section{Alcune conclusioni}

La riflessione dottrinale che si è tentato di sintetizzare nelle pagine che precedono è nel complesso molto ricca ed è dotata sia di profondità storica che di apertura alle riflessioni di altre culture giuridiche, oltre che ai dati provenienti dal diritto europeo, che - come si è visto - ha svolto un ruolo decisivo sui temi qui accennati. L'interpretazione estensiva - e forse anche evolutiva - dei diritti fondamentali di libertà di comunicazione e di espressione, da un lato, e le categorie del diritto europeo in materia di protezione dei dati personali offrono le basi più importanti attraverso le quali la cultura giuridica italiana ha tentato di rielaborare il tema del rapporto fra diritti fondamentali e nuove tecnologie.

Si tratta, ovviamente, di uno scenario in movimento, dato che la rivoluzione informatica non sembra ancora aver raggiunto un punto di approdo: anzi, alcune questioni, come quelle poste nel campo dell'intelligenza artificiale, stanno appena iniziando a manifestare la loro portata e gli effetti, che per ora è soltanto possibile intravedere, appaiono così devastanti da richiedere, nel prossimo futuro, la costruzione di categorie giuridiche interamente nuove. Talmente nuove da richiedere, forse, un vero e proprio sforzo «costituente», dato che alcuni cambiamenti tecnologici rischiano di alterare la struttura fondamentale delle società tecnologicamente avanzate $\mathrm{e}$, con esse, sia il modo in cui tali società sono governate o si autogovernano, sia i diritti fondamentali della persona. 